\title{
FAMILIES OF FRACTIONAL CAUCHY TRANSFORMS IN THE BALL
}

\author{
E. S. DUBTSOV
}

Dedicated to Victor Petrovich Havin on the occasion of his 75th birthday

\begin{abstract}
Let $B_{n}$ denote the unit ball in $\mathbb{C}^{n}, n \geq 1$. Given $\alpha>0$, let $\mathcal{K}_{\alpha}(n)$ denote the class of functions defined for $z \in B_{n}$ by integrating the kernel $(1-\langle z, \zeta\rangle)^{-\alpha}$ against a complex-valued Borel measure on the sphere $\left\{\zeta \in \mathbb{C}^{n}:|\zeta|=1\right\}$. The families $\mathcal{K}_{\alpha}(1)$ of fractional Cauchy transforms have been investigated intensively by several authors. In the paper, various properties of $\mathcal{K}_{\alpha}(n), n \geq 2$, are studied. In particular, relations between $\mathcal{K}_{\alpha}(n)$ and other spaces of holomorphic functions in the ball are obtained. Also, pointwise multipliers for the spaces $\mathcal{K}_{\alpha}(n)$ are investigated.
\end{abstract}

\section{§1. INTRODUCTION}

For $n \geq 1$, put $B_{n}=\left\{z \in \mathbb{C}^{n}:|z|<1\right\}$. Let $M(n)$ denote the space of complexvalued Borel measures on the sphere $\partial B_{n}$.

1.1. Fractional Cauchy transforms. Let $\alpha>0$. Given a measure $\mu \in M(n)$, its fractional Cauchy transform of order $\alpha$ is defined by the identity

$$
K_{\alpha}[\mu](z)=\int_{\partial B_{n}} \frac{1}{(1-\langle z, \zeta\rangle)^{\alpha}} d \mu(\zeta), \quad z \in B_{n} .
$$

Here and in what follows we use the principal branch of the logarithm. Put

$$
\mathcal{K}_{\alpha}(n)=\left\{K_{\alpha}[\mu]: \mu \in M(n)\right\} .
$$

1.2. Multipliers. Let $\mathcal{H o l}\left(B_{n}\right)$ denote the space of holomorphic functions in the ball $B_{n}$. A function $g \in \mathcal{H o l}\left(B_{n}\right)$ is called a multiplier for the family $\mathcal{K}_{\alpha}(n), \alpha>0$, if $f g \in \mathcal{K}_{\alpha}(n)$ for all $f \in \mathcal{K}_{\alpha}(n)$. Let $\mathfrak{M}_{\alpha}(n)$ denote the set of all multipliers for $\mathcal{K}_{\alpha}(n)$.

1.3. Families $\mathcal{K}_{\alpha}(n)$ and $\mathfrak{M}_{\alpha}(n)$ as Banach spaces. For $f \in \mathcal{K}_{\alpha}(n)$, put

$$
\|f\|_{\mathcal{K}_{\alpha}(n)}=\inf \left\{\|\mu\|_{M(n)}: f=K_{\alpha}[\mu]\right\} .
$$

Standard arguments show that the above infimum is attained, and that $\mathcal{K}_{\alpha}(n)$ with the norm $\|\cdot\|_{\mathcal{K}_{\alpha}(n)}$ is a Banach space. Next, assume that $f=K_{\alpha}[\rho]$, where $\rho$ is a positive measure. For $\mu \in M(n)$, let $f=K_{\alpha}[\mu]$; then $\|\rho\|=K_{\alpha}[\rho](0)=K_{\alpha}[\mu](0) \leq\|\mu\|$. Therefore, $\|f\|_{\mathcal{K}_{\alpha}(n)}=\|\rho\|_{M(n)}$.

For $g \in \mathfrak{M}_{\alpha}(n)$, put

$$
\|g\|_{\mathfrak{M}_{\alpha}(n)}=\sup \left\{\|f g\|_{\mathcal{K}_{\alpha}(n)}:\|f\|_{\mathcal{K}_{\alpha}(n)} \leq 1\right\} .
$$

The closed graph theorem guarantees that $\|g\|_{\mathfrak{M}_{\alpha}(n)}<\infty$. The space $\mathfrak{M}_{\alpha}(n)$ with the norm $\|\cdot\|_{\mathfrak{M}_{\alpha}(n)}$ is a Banach space. Also, note that $\mathfrak{M}_{\alpha}(n)$ is a Banach algebra.

2010 Mathematics Subject Classification. Primary 32A26, 32A37.

Key words and phrases. Fractional Cauchy transform, Bergman-Sobolev space, pointwise multiplier. Supported by RFBR (grant no. 08-01-00358-a) and by the Russian Science Support Foundation. 
1.4. Historical remarks. The families $\mathcal{K}_{\alpha}(n)$ generalize the classical family $\mathcal{K}_{n}(n)$ of Cauchy integrals. Investigations of the family $\mathcal{K}_{1}(1)$ as a Banach space were initiated by Havin in [14, 15]. Further results and references are presented in the monograph $[9$. The spaces $\mathcal{K}_{\alpha}(1), \alpha>0$, were introduced by MacGregor [20]. Various properties of the families $\mathcal{K}_{\alpha}(1)$ are collected in [17. In the present paper we focus on the study of the families $\mathcal{K}_{\alpha}(n)$ with $n \geq 2$. Certain properties of the spaces $\mathcal{K}_{n}(n), n \in \mathbb{N}$, were proved in the survey [3]. To the best knowledge of the author, for $n \geq 2$ the families $\mathcal{K}_{\alpha}(n)$ have not been investigated systematically.

1.5. Related problems. Let $\sigma_{n}$ denote the normalized Lebesgue measure on the sphere $\partial B_{n}$. For $1 \leq p \leq \infty$ and $0<\alpha<n$, put

$$
\mathcal{K}_{\alpha}^{p}(n)=\left\{K_{\alpha}\left[f \sigma_{n}\right]: f \in L^{p}\left(\sigma_{n}\right)\right\} .
$$

The boundary behavior of functions belonging to $\mathcal{K}_{\alpha}^{p}(1)$ was investigated in [21], where the families $\mathcal{K}_{\alpha}^{p}(1)$ were called Dirichlet type spaces. The families $\mathcal{K}_{\alpha}^{p}(n), n \in \mathbb{N}$, were investigated in [26]; see also [1, 19, 8].

1.6. Organization of the paper. Embedding properties for $\mathcal{K}_{\alpha}(n)$ and $\mathfrak{M}_{\alpha}(n)$ are studied in \$2 Relations between $\mathcal{K}_{\alpha}(n)$ and the classical spaces of holomorphic functions are obtained in $\S \S 3$ and 5 . Also, in $\S \S 4$ and 5 we study differentiation operators on the spaces $\mathcal{K}_{\alpha}(n)$. The boundary behavior of the fractional Cauchy transforms is investigated in 96 . Finally, some results about the multiplier spaces $\mathfrak{M}_{\alpha}(n)$ are obtained in 97 .

\section{§2. EMbedding PROPERTIES}

2.1. Embedding properties for families of fractional Cauchy transforms. Below we shall use the following lemma.

Lemma 2.1 (see [7, Lemma 1]). Assume that $u, v \in B_{1}, \alpha>0, \beta>0$. Then

$$
(1-u)^{-\alpha}(1-v)^{-\beta}=\frac{\Gamma(\alpha+\beta)}{\Gamma(\alpha) \Gamma(\beta)} \int_{0}^{1} t^{\alpha-1}(1-t)^{\beta-1}[1-(t u+(1-t) v)]^{-\alpha-\beta} d t .
$$

Let $\mathcal{P}(n)$ denote the set of all probability measures on the sphere $\partial B_{n}, n \in \mathbb{N}$. For $n=1$, the following result was obtained in [7].

Proposition 2.2. Put $\mathcal{F}_{\alpha}(n)=\left\{K_{\alpha}[\mu]: \mu \in \mathcal{P}(n)\right\}, n \in \mathbb{N}$.

(i) If $\alpha>0$ and $\beta>0$, then $\mathcal{F}_{\alpha}(n) \cdot \mathcal{F}_{\beta}(n) \subset \mathcal{F}_{\alpha+\beta}(n)$.

(ii) If $0<\alpha<\beta$, then $\mathcal{F}_{\alpha}(n) \subset \mathcal{F}_{\beta}(n)$.

Proof. First, we show how to deduce statement (i) from the following property:

$$
(1-\langle z, \zeta\rangle)^{-\alpha}(1-\langle z, \xi\rangle)^{-\beta} \in \mathcal{F}_{\alpha+\beta}(n), \quad z \in B_{n},
$$

for any fixed points $\zeta, \xi \in \partial B_{n}$. So, let $f \in \mathcal{F}_{\alpha}(n)$, and let $g \in \mathcal{F}_{\beta}(n)$. Then, by the definition,

$$
f(z) g(z)=\int_{\partial B_{n} \times \partial B_{n}}(1-\langle z, \zeta\rangle)^{-\alpha}(1-\langle z, \xi\rangle)^{-\beta} d \rho(\zeta, \xi), \quad z \in B_{n},
$$

where $\rho$ is a probability measure on the product $\partial B_{n} \times \partial B_{n}$. We approximate the measure $\rho$ in the weak* topology by probability measures $\rho_{j}$, where every $\rho_{j}$ is a finite sum of atomic charges. The set $\mathcal{F}_{\alpha+\beta}(n)$ is convex; therefore, on the one hand, we have

$$
\int_{\partial B_{n} \times \partial B_{n}}(1-\langle z, \zeta\rangle)^{-\alpha}(1-\langle z, \xi\rangle)^{-\beta} d \rho_{j}(\zeta, \xi) \in \mathcal{F}_{\alpha+\beta}(n) .
$$


On the other hand, without loss of generality we may assume that the convergence

$$
\int_{\partial B_{n} \times \partial B_{n}}(1-\langle z, \zeta\rangle)^{-\alpha}(1-\langle z, \xi\rangle)^{-\beta} d \rho_{j}(\zeta, \xi) \rightarrow f(z) g(z)
$$

as $j \rightarrow \infty$ is uniform on the compact subsets of the ball $B_{n}$. It remains to observe that the set $\mathcal{F}_{\alpha+\beta}(n)$ is closed in the topology of uniform convergence on compact subsets of the ball.

Now, we prove property (2.1). Lemma 2.1 guarantees that

$$
(1-\langle z, \zeta\rangle)^{-\alpha}(1-\langle z, \xi\rangle)^{-\beta}=\frac{\Gamma(\alpha+\beta)}{\Gamma(\alpha) \Gamma(\beta)} \int_{0}^{1} t^{\alpha-1}(1-t)^{\beta-1}[1-\langle z, w(t)\rangle]^{-\alpha-\beta} d t
$$

where $z \in B_{n}$ and $w(t)=t \zeta+(1-t) \xi$. Since

$$
\Gamma(\alpha+\beta) \Gamma(\alpha)^{-1} \Gamma(\beta)^{-1} t^{\alpha-1}(1-t)^{\beta-1} d t
$$

is a probability measure on the interval $[0,1]$, it suffices to show that

$$
[1-\langle z, w(t)\rangle]^{-\alpha-\beta} \in \mathcal{F}_{\alpha+\beta}(n), \quad z \in B_{n}
$$

for every fixed parameter $t \in[0,1]$. If $t=0$ or $t=1$, then property (2.3) holds true by definition. So, assume that $0<t<1$. Then $w(t) \in B_{n}$. Next, note that $[1-\langle z, \cdot\rangle]^{-\alpha-\beta}$ is a harmonic function in the closed ball $\bar{B}_{n}$. Hence,

$$
\frac{1}{[1-\langle z, w\rangle]^{\alpha+\beta}}=\int_{\partial B_{n}} \frac{1-|w|^{2}}{|w-\eta|^{2 n}} \frac{1}{[1-\langle z, \eta\rangle]^{\alpha+\beta}} d \sigma_{n}(\eta)
$$

If a point $w \in B_{n}$ is fixed, then $\left(1-|w|^{2}\right)|w-\eta|^{-2 n} d \sigma_{n}(\eta)$ is a probability measure. Thus, to prove (2.3), it suffices to put $w=w(t), 0<t<1$. The proof of property (i) is finished.

Let $\beta>\alpha>0$. Recall that the ball algebra $A\left(B_{n}\right)$ is defined by

$$
A\left(B_{n}\right)=\mathcal{H o l}\left(B_{n}\right) \cap C\left(\overline{B_{n}}\right) .
$$

If $z \in B_{n}$, then $(1-\langle z, \cdot\rangle)^{-\beta+\alpha} \in \bar{A}\left(B_{n}\right)$. Hence,

$$
1=\int_{\partial B_{n}} \frac{1}{(1-\langle z, \zeta\rangle)^{\beta-\alpha}} d \sigma_{n}(\zeta), \quad z \in B_{n} .
$$

In other words, $1 \in \mathcal{F}_{\beta-\alpha}(n)$; thus, (i) implies (ii).

Corollary 2.3. Let $n \in \mathbb{N}$.

(i) If $\alpha>0$ and $\beta>0$, then $\mathcal{K}_{\alpha}(n) \cdot \mathcal{K}_{\beta}(n) \subset \mathcal{K}_{\alpha+\beta}(n)$.

(ii) If $0<\alpha<\beta$, then $\mathcal{K}_{\alpha}(n) \subset \mathcal{K}_{\beta}(n)$.

Proof. Let $f=K_{\alpha}[\mu]$ and let $g=K_{\beta}[\rho]$, where $\mu, \rho \in M(n)$ and $\alpha, \beta>0$. Considering the Jordan decompositions of the measures $\mu, \rho$ and applying part (i) of Proposition 2.2, we obtain properties (i) and (ii).

2.2. Embedding properties for the multiplier families. The proof of the following lemma will be omitted, because it practically coincides with that given in 29] for the case where $n=\alpha=1$ (see also [16], where the case of $n=1, \alpha>0$ was considered).

Lemma 2.4. Assume that $n \in \mathbb{N}$ and $\alpha>0$. Then the following properties are equivalent:

(i) $g \in \mathfrak{M}_{\alpha}(n)$;

(ii) $g(z)(1-\langle z, \zeta\rangle)^{-\alpha} \in \mathcal{K}_{\alpha}(n)$ for all $\zeta \in \partial B_{n}$ and

$$
\sup \left\{\left\|\frac{g(z)}{(1-\langle z, \zeta\rangle)^{\alpha}}\right\|_{\mathcal{K}_{\alpha}(n)}: \zeta \in \partial B_{n}\right\}<\infty .
$$


For $n=1$, the following proposition was proved in [16].

Proposition 2.5. If $n \in \mathbb{N}$ and $0<\alpha<\beta$, then $\mathfrak{M}_{\alpha}(n) \subset \mathfrak{M}_{\beta}(n)$.

Proof. Let $g \in \mathfrak{M}_{\alpha}(n)$. By Lemma 2.4 there exists a constant $C>0$ and there exist measures $\mu_{\zeta} \in M(n), \zeta \in \partial B_{n}$, such that $\left\|\mu_{\zeta}\right\| \leq C$ and

$$
\frac{g(z)}{(1-\langle z, \zeta\rangle)^{\alpha}}=\int_{\partial B_{n}} \frac{1}{(1-\langle z, \xi\rangle)^{\alpha}} d \mu_{\zeta}(\xi), \quad z \in B_{n} .
$$

Hence,

$$
\frac{g(z)}{(1-\langle z, \zeta\rangle)^{\beta}}=\int_{\partial B_{n}} \frac{1}{(1-\langle z, \xi\rangle)^{\alpha}} \frac{1}{(1-\langle z, \zeta\rangle)^{\beta-\alpha}} d \mu_{\zeta}(\xi) .
$$

By (2.2) and (2.4), for every pair of points $\zeta, \xi \in \partial B_{n}$, there exists a probability measure $\rho_{\zeta, \xi} \in M(n)$ such that

$$
\frac{1}{(1-\langle z, \xi\rangle)^{\alpha}} \frac{1}{(1-\langle z, \zeta\rangle)^{\beta-\alpha}}=\int_{\partial B_{n}} \frac{1}{(1-\langle z, \eta\rangle)^{\beta}} d \rho_{\zeta, \xi}(\eta) .
$$

Therefore,

$$
\frac{g(z)}{(1-\langle z, \zeta\rangle)^{\beta}}=\int_{\partial B_{n}} \int_{\partial B_{n}} \frac{1}{(1-\langle z, \eta\rangle)^{\beta}} d \rho_{\zeta, \xi}(\eta) d \mu_{\zeta}(\xi) .
$$

Fix a point $\zeta \in \partial B_{n}$. We approximate the measure $\mu=\mu_{\zeta}$ in the weak ${ }^{*}$ topology by measures $\mu_{k}$ such that $\left\|\mu_{k}\right\| \leq C$ and $\mu_{k}=\sum_{j=1}^{J(k)} a_{j, k} \delta_{\xi_{j, k}}, a_{j, k} \in \mathbb{C}$. Let $\lambda=\lambda_{\zeta}$ denote an accumulation point of the sequence $\lambda_{k}=\sum_{j=1}^{J(k)} a_{j, k} \rho_{\zeta, \xi_{j, k}}$ in the weak* topology. Then

$$
\frac{g(z)}{(1-\langle z, \zeta\rangle)^{\beta}}=\int_{\partial B_{n}} \frac{1}{(1-\langle z, \eta\rangle)^{\beta}} d \lambda_{\zeta}(\eta)
$$

where $\left\|\lambda_{\zeta}\right\| \leq C$. Thus, Lemma 2.4 guarantees that $g \in \mathfrak{M}_{\beta}(n)$.

\section{§3. Fractional Cauchy transforms and Hardy spaces}

For $f \in \mathcal{H o l}\left(B_{n}\right), 0<p<+\infty$ and $0<r<1$, put

$$
M_{p}(f, r)=\left(\int_{\partial B_{n}}|f(r \zeta)|^{p} d \sigma_{n}(\zeta)\right)^{\frac{1}{p}} .
$$

Therefore, the classical Hardy space $H^{p}\left(B_{n}\right)$ is defined by

$$
H^{p}\left(B_{n}\right)=\left\{f \in \mathcal{H} \text { ol }\left(B_{n}\right): M_{p}(f, r) \leq C\right\} .
$$

Recall that if $f$ and $p$ are fixed, then the integral mean $M_{p}(f, r)$ is a monotone increasing function of the variable $r \in(0,1)$.

For $n \in \mathbb{N}$, let $\mathcal{K}_{0}(n)$ denote the family of all functions $f$ such that

$$
f(z)-f(0)=\int_{\partial B_{n}} \log \frac{1}{1-\langle z, \zeta\rangle} d \mu(\zeta), \quad z \in B_{n},
$$

for a measure $\mu \in M(n)$.

Let $f \in \mathcal{K}_{\alpha}(n), \alpha \geq 0$. In the present section we study the growth of the integral means $M_{p}(f, r)$ as $r \rightarrow 1-$. For $n=1$, the corresponding results were obtained in [11].

The following lemma will be used below in numerous situations. The notation $a(z) \approx$ $b(z)$ means that the quotient $a(z) / b(z)$ has a finite positive limit as $|z| \rightarrow 1-$.

Lemma 3.1 (see [23, Proposition 1.4.10]). Suppose $n \in \mathbb{N}$ and $c \in \mathbb{R}$. Put

$$
I_{c}(z)=\int_{\partial B_{n}} \frac{d \sigma_{n}(\zeta)}{\mid 1-\left\langle z,\left.\zeta\right|^{n+c}\right.}, \quad z \in \bar{B}_{n}
$$


(i) If $c<0$, then the function $I_{c}(z)$ is bounded in the closed ball $\bar{B}_{n}$.

(ii) If $c>0$, then $I_{c}(z) \approx\left(1-|z|^{2}\right)^{-c}$.

(iii) Finally,

$$
I_{0}(z) \approx \log \frac{1}{\left(1-|z|^{2}\right)} .
$$

Proposition 3.2. For $\alpha \geq 0$, let $f \in \mathcal{K}_{\alpha}(n)$. The following estimates hold true as $r \rightarrow 1-$.

If $\alpha=0$ and $0<p<+\infty$, then

$$
M_{p}^{p}(f, r)=\mathcal{O}(1) .
$$

If $0<\alpha \leq n$ and $0<p<n / \alpha$, then

$$
M_{p}^{p}(f, r)=\mathcal{O}(1) .
$$

If $0<\alpha \leq n$ and $p=n / \alpha$, then

$$
M_{p}^{p}(f, r)=\mathcal{O}\left(\log \frac{1}{1-r}\right)
$$

If $p>n / \alpha$ and $p \geq 1$, then

$$
M_{p}^{p}(f, r)=\mathcal{O}\left[\frac{1}{(1-r)^{\alpha p-n}}\right] .
$$

If $0<p<1$ and $\alpha \geq 2 n$, then

$$
M_{p}^{p}(f, r)=\mathcal{O}\left[\frac{1}{(1-r)^{(\alpha-n) p}}\right] .
$$

Proof. It is well known that $\mathcal{K}_{n}(n) \subset H^{p}\left(B_{n}\right)$ for all $0<p<1$ [23, Theorem 6.2.3]. In other words, estimate (3.3) is fulfilled for $\alpha=n$. Next, let $f=K_{\alpha}[\mu]$.

Assume that $p \geq 1$. Then the integral Minkowski inequality, Fubini's theorem, and Lemma 3.1 guarantee that

$$
\begin{aligned}
M_{p}^{p}(f, r)=\int_{\partial B_{n}}|f(r \xi)|^{p} d \sigma_{n}(\xi) \leq & \int_{\partial B_{n}} \int_{\partial B_{n}} \frac{1}{|1-\langle r \xi, \zeta\rangle|^{\alpha p}} d \sigma_{n}(\xi) d|\mu|(\zeta) \\
= & \begin{cases}\mathcal{O}(1) & \text { if } \alpha p<n, \\
\mathcal{O}\left(\log \frac{1}{1-r}\right) & \text { if } \alpha p=n, \\
\mathcal{O}\left[\frac{1}{(1-r)^{\alpha p-n}}\right] & \text { if } \alpha p>n .\end{cases}
\end{aligned}
$$

This proves estimates (3.4) and (3.5). Property (3.3) is proved for $p \geq 1$. Recall that $H^{q}\left(B_{n}\right) \subset H^{t}\left(B_{n}\right)$ for $q>t>0$. Hence, estimate (3.3) is valid for all $0<p<n / \alpha$.

If $\gamma>0$, then

$$
-\log 2 \leq \log \frac{1}{|1-\langle r \xi, \zeta\rangle|} \leq C(\gamma) \frac{1}{|1-\langle r \xi, \zeta\rangle|^{\gamma}},
$$

where $0<r<1$ and $\xi, \zeta \in \partial B_{n}$. Hence, estimate (3.2) is proved for all $p \in(0,+\infty)$.

Now, assume that $0<p<1$ and $\alpha \geq 2 n$. Note that $(a+b)^{p} \leq C(p)\left(a^{p}+b^{p}\right)$ for $a \geq 0, b \geq 0$. Hence, by the Jordan decomposition theorem, we may assume that $\mu$ is a probability measure. Therefore, we obtain

$$
\begin{aligned}
|f(z)| & \leq \int_{\partial B_{n}} \frac{1}{|1-\langle z, \zeta\rangle|^{\alpha}} d \mu(\zeta) \\
& \leq \frac{1}{(1-|z|)^{\alpha-2 n}} \int_{\partial B_{n}} \frac{1}{|1-\langle z, \zeta\rangle|^{2 n}} d \mu(\zeta) \\
& =\frac{1}{(1-|z|)^{\alpha-n}} \int_{\partial B_{n}} \frac{\left(1-|z|^{2}\right)^{n}}{|1-\langle z, \zeta\rangle|^{2 n}} d \mu(\zeta) .
\end{aligned}
$$


Note that

$$
u(z)=\int_{\partial B_{n}} \frac{\left(1-|z|^{2}\right)^{n}}{\mid 1-\langle z, \zeta\rangle^{2 n}} d \mu(\zeta)
$$

is a positive $\mathcal{M}$-harmonic function in the ball. Thus,

$$
\int_{\partial B_{n}} u(r \zeta) d \sigma_{n}(\zeta)=u(0)
$$

for $0<r<1$; see [23, Theorem 4.2.4]. Next, since $1 / p>1$, Minkowski's inequality yields

$$
\left(\int_{\partial B_{n}} u^{p}(r \zeta) d \sigma_{n}(\zeta)\right)^{\frac{1}{p}} \leq \int_{\partial B_{n}} u^{p \cdot \frac{1}{p}}(r \zeta) d \sigma_{n}(\zeta)=u(0)
$$

for $0<r<1$.

Estimates (3.7) and (3.8) imply that

$$
M_{p}^{p}(f, r)=\int_{\partial B_{n}}|f(r \zeta)|^{p} d \sigma_{n}(\zeta) \leq \frac{1}{(1-r)^{(\alpha-n) p}} u^{p}(0)=\frac{\left(\mu\left(\partial B_{n}\right)\right)^{p}}{(1-r)^{(\alpha-n) p}} .
$$

This proves (3.6).

Corollary 3.3. Let $n \in \mathbb{N}$.

(i) If $0 \leq \alpha \leq n$, then $\mathcal{K}_{\alpha}(n) \subset H^{p}\left(B_{n}\right)$ for $0<p<n / \alpha$.

(ii) If $f \in H^{1}\left(B_{n}\right)$, then $f \in \mathcal{K}_{n}(n)$; moreover, $\|f\|_{\mathcal{K}_{n}(n)} \leq\|f\|_{H^{1}\left(B_{n}\right)}$.

(iii) $\mathcal{K}_{0}(n) \subset \mathcal{K}_{n}(n)$.

Proof. Part (i) is equivalent to estimates (3.2) and (3.3). Next, if $f \in H^{1}\left(B_{n}\right)$, then $f=K_{n}\left[f^{*}\right]$, where $f^{*}(\zeta)=\lim _{r \rightarrow 1-} f(r \zeta), \zeta \in \partial B_{n}$. Therefore, $\|f\|_{\mathcal{K}_{n}(n)} \leq\|f\|_{H^{1}\left(B_{n}\right)}$. Finally, part (iii) follows from (i) and (ii).

Part (iii) of Corollary 3.3 will be improved in Corollary 5.5. Also, estimate (3.3) can be refined in terms of the weak Hardy spaces $H^{p, \infty}\left(B_{n}\right)$. Let $p>0$. By definition, $f \in H^{p, \infty}\left(B_{n}\right)$ if $f \in H^{q}\left(B_{n}\right)$ for some $q>0$ and

$$
\|f\|_{p, \infty}^{p}=\sup _{t>0} t^{p} \sigma_{n}\left\{\zeta \in \partial B_{n}:\left|f^{*}(\zeta)\right|>t\right\}<\infty .
$$

Proposition 3.4. If $0<\alpha \leq n$, then $\mathcal{K}_{\alpha}(n) \subset H^{\frac{n}{\alpha}, \infty}\left(B_{n}\right)$.

Proof. The argument below is due to A. B. Aleksandrov. The case of $\alpha=n$ is well known. So, assume that $p=n / \alpha>1$; there exists a norm $\|\cdot\|_{p}^{*}$ on the space $H^{p, \infty}\left(B_{n}\right)$ such that

$$
\|f\|_{p, \infty} \leq C_{1}(p)\|f\|_{p}^{*} \leq C_{2}(p)\|f\|_{p, \infty}
$$

for all $f \in H^{p, \infty}\left(B_{n}\right)$, for some positive constants $C_{1}(p)$ and $C_{2}(p)$ (see, e.g., [6]).

Now, assume that $f \in K_{\alpha}[\mu], \mu \in M(n)$. We must prove that $f \in H^{p, \infty}\left(B_{n}\right)$. Suppose, without loss of generality, that $\mu$ is a probability measure. We approximate the measure $\mu$ in the weak ${ }^{*}$ topology by probability measures $\mu_{k}=\sum_{j=1}^{J(k)} a_{k, j} \delta_{\xi_{k, j}}$, where $a_{k, j}>0$ and $\delta_{\xi_{k, j}}$ denotes the $\delta$-measure at the point $\xi_{k, j} \in \partial B_{n}$. Applying estimates (3.9), the triangle inequality, and Proposition 5.1.4 in [23], we obtain

$$
\begin{aligned}
\left\|K_{\alpha}\left[\mu_{k}\right]\right\|_{p, \infty} & \leq C_{1}(p)\left\|K_{\alpha}\left[\mu_{k}\right]\right\|_{p}^{*} \leq C_{1}(p)\left\|\left(1-z_{1}\right)^{-\alpha}\right\|_{p}^{*} \\
& \leq C_{2}(p)\left\|\left(1-z_{1}\right)^{-\alpha}\right\|_{p, \infty} \leq C(p, n) .
\end{aligned}
$$

Finally, since $\mu_{k} \rightarrow \mu$ in the weak* topology, we see that $f \in H^{p, \infty}\left(B_{n}\right)$. The proof is finished. 


\section{$\S 4$. FAMilies $\mathcal{K}_{\alpha}(n)$ AND Differentiation}

Let $f \in \mathcal{H o l}\left(B_{1}\right)$, and let $\alpha \geq 0$. It is well known that $f \in \mathcal{K}_{\alpha}(1)$ if and only if $f^{\prime} \in \mathcal{K}_{\alpha+1}(1)$. In the present section, we prove similar assertions for $f \in \mathcal{H} \operatorname{Hol}\left(B_{n}\right)$, $n \in \mathbb{N}$.

4.1. Radial derivatives. Let $f \in \mathcal{H}$ ol $\left(B_{n}\right)$. The radial derivative $\mathcal{R} f$ is defined by the identity

$$
\mathcal{R} f(z)=\sum_{j=1}^{n} z_{j} \frac{\partial f}{\partial z_{j}}(z) .
$$

Recall that

$$
f(z)-f(0)=\int_{0}^{1} \frac{\mathcal{R} f(t z)}{t} d t, \quad z \in B_{n} .
$$

Proposition 4.1. Let $f \in \mathcal{H}$ ol $\left(B_{n}\right), n \in \mathbb{N}$. Then $f \in \mathcal{K}_{0}(n)$ if and only if $\mathcal{R} f \in \mathcal{K}_{1}(n)$.

Proof. Suppose that $f \in \mathcal{K}_{0}(n)$, that is, (3.1) is true. Then

$$
\mathcal{R} f(z)=\int_{\partial B_{n}} \frac{\langle z, \zeta\rangle}{1-\langle z, \zeta\rangle} d \mu(\zeta)=K_{1}[\rho](z)
$$

where $\rho=\mu-\mu\left(\partial B_{n}\right) \sigma_{n}$.

To prove the reverse implication, assume that

$$
\mathcal{R} f(z)=\int_{\partial B_{n}} \frac{1}{1-\langle z, \zeta\rangle} d \rho(\zeta)
$$

where $\rho \in M(n)$. Note that $\mathcal{R} f(0)=0$; thus, $\rho\left(\partial B_{n}\right)=0$. Applying identity (4.1) and the property $\rho\left(\partial B_{n}\right)=0$, we obtain

$$
\begin{aligned}
f(z)-f(0) & =\int_{0}^{1} \int_{\partial B_{n}} \frac{1}{t(1-t\langle z, \zeta\rangle)} d \rho(\zeta) d t \\
& =\int_{0}^{1} \int_{\partial B_{n}}\left(\frac{1}{t}+\frac{\langle z, \zeta\rangle}{1-t\langle z, \zeta\rangle}\right) d \rho(\zeta) d t \\
& =\int_{\partial B_{n}} \int_{0}^{1} \frac{\langle z, \zeta\rangle}{1-t\langle z, \zeta\rangle} d t d \rho(\zeta) \\
& =\int_{\partial B_{n}} \log \frac{1}{1-\langle z, \zeta\rangle} d \rho(\zeta)
\end{aligned}
$$

as required.

Proposition 4.2. Assume that $\alpha>0$ and $f \in \mathcal{H}$ ol $\left(B_{n}\right), n \in \mathbb{N}$. Then $f \in \mathcal{K}_{0}(n)+\mathcal{K}_{\alpha}(n)$ if and only if $\mathcal{R} f \in \mathcal{K}_{\alpha+1}(n)$.

Proof. Let $g \in \mathcal{K}_{0}(n)$ and $h \in \mathcal{K}_{\alpha}(n)$, that is,

$$
h(z)=\int_{\partial B_{n}} \frac{1}{(1-\langle z, \zeta\rangle)^{\alpha}} d \mu(\zeta), \quad z \in B_{n} .
$$

Straightforward calculations show that

$$
\mathcal{R} h(z)=\int_{\partial B_{n}} \frac{\alpha}{(1-\langle z, \zeta\rangle)^{\alpha+1}} d \mu(\zeta)-\alpha h(z) \in \mathcal{K}_{\alpha+1}(n)
$$

because $\alpha h \in \mathcal{K}_{\alpha}(n) \subset \mathcal{K}_{\alpha+1}(n)$ by part (ii) of Corollary 2.3. On the other hand, Proposition 4.1 guarantees that $\mathcal{R} g \in \mathcal{K}_{1}(n) \subset \mathcal{K}_{\alpha+1}(n)$.

It is convenient to split the proof of the reverse implication into two steps. 
Step 1. Let $\alpha=m \in \mathbb{N}$. By the hypothesis, we have

$$
\mathcal{R} f(z)=\int_{\partial B_{n}} \frac{1}{(1-\langle z, \zeta\rangle)^{m+1}} d \mu(\zeta), \quad z \in B_{n} .
$$

Hence,

$$
f(z)-f(0)=\int_{0}^{1} \frac{\mathcal{R} f(t z)}{t} d t=\int_{0}^{1} \int_{\partial B_{n}} \frac{1}{t(1-t\langle z, \zeta\rangle)^{m+1}} d \mu(\zeta) d t, \quad z \in B_{n} .
$$

If $w \in \mathbb{C}$ and $|w|<1$, then

$$
\frac{1}{t(1-t w)^{m+1}}=\frac{1}{t}+\sum_{j=1}^{m+1} \frac{w}{(1-t w)^{j}} .
$$

Since $\mathcal{R} f(0)=0$, we have $\mu\left(\partial B_{n}\right)=0$. Putting $w=\langle z, \zeta\rangle$, we obtain

$$
\begin{aligned}
f(z)-f(0) & =\sum_{j=1}^{m+1} \int_{\partial B_{n}} \int_{0}^{1} \frac{\langle z, \zeta\rangle}{(1-t\langle z, \zeta\rangle)^{j}} d t d \mu(\zeta) \\
& =-\int_{\partial B_{n}} \log (1-\langle z, \zeta\rangle) d \mu(\zeta)+\sum_{j=2}^{m+1} \int_{\partial B_{n}} \frac{1}{(j-1)(1-\langle z, \zeta\rangle)^{j-1}} d \mu(\zeta) .
\end{aligned}
$$

The embeddings $\mathcal{K}_{j-1}(n) \subset \mathcal{K}_{m}(n), j=2, \ldots, m$, guarantee that $f \in \mathcal{K}_{0}(n)+\mathcal{K}_{m}(n)$.

Step 2. Let $\alpha>0, \alpha \notin \mathbb{N}$. Repeating the arguments of Step 1 and reversing the order of integration, we have

$$
\begin{aligned}
f(z)-f(0) & =\int_{0}^{1} \int_{\partial B_{n}} \frac{1}{t(1-t\langle z, \zeta\rangle)^{\alpha+1}} d \mu(\zeta) d t \\
& =\int_{\partial B_{n}} \int_{0}^{1} \frac{\langle z, \zeta\rangle}{(1-t\langle z, \zeta\rangle)^{\alpha+1}} d t d \mu(\zeta)+\int_{0}^{1} \frac{1}{t} \int_{\partial B_{n}} \frac{1}{(1-t\langle z, \zeta\rangle)^{\alpha}} d \mu(\zeta) d t .
\end{aligned}
$$

The inner integral in the first summand can be calculated explicitly. So, consider the second summand. Put $[\alpha]=m \in \mathbb{N} \cup\{0\}$. Note that $m+1>\alpha$ and $\mu\left(\partial B_{n}\right)=0$. Hence, by part (ii) of Corollary 2.3 , there exists a measure $\rho \in M(n)$ such that $K_{m+1}[\rho]=K_{\alpha}[\mu]$. Also, we have $\rho\left(\partial B_{n}\right)=K_{m+1}[\rho](0)=K_{\alpha}[\mu](0)=\mu\left(\partial B_{n}\right)=0$. Therefore, reversing the order of integration once again, we obtain

$$
f(z)-f(0)=\frac{1}{\alpha} \int_{\partial B_{n}} \frac{1}{(1-\langle z, \zeta\rangle)^{\alpha}} d \mu(\zeta)+\int_{\partial B_{n}} \int_{0}^{1} \frac{1}{t(1-t\langle z, \zeta\rangle)^{m+1}} d t d \rho(\zeta) .
$$

By the definition, the first summand in the above sum belongs to $\mathcal{K}_{\alpha}(n)$. If $m=0$, then the proof of Proposition 4.1 shows that the second summand is in the family $\mathcal{K}_{0}(n)$. Finally, for $m \in \mathbb{N}$, at Step 1 it was proved that the second summand belongs to $\mathcal{K}_{0}(n)+$ $\mathcal{K}_{m}(n) \subset \mathcal{K}_{0}(n)+\mathcal{K}_{\alpha}(n)$.

Note that Proposition 4.2 admits refinement (see Corollary [5.6).

4.2. Fractional differential operators of order 1. By definition, we put $R^{1} f=$ $f+\mathcal{R} f$ for $f \in \mathcal{H} \operatorname{ol}\left(B_{n}\right)$. The operator $R^{1}$ is called the fractional differential operator of order 1 . Observe that

$$
f(z)=\int_{0}^{1} R^{1} f(t z) d t, \quad z \in B_{n} .
$$

The following proposition can be proved with the help of elementary methods. 
Proposition 4.3. Let $f \in \mathcal{H}$ ol $\left(B_{n}\right), n \in \mathbb{N}$.

(i) If $\alpha>0$, then the property $f \in \mathcal{K}_{\alpha}(n)$ implies that $R^{1} f \in \mathcal{K}_{\alpha+1}(n)$.

(ii) If $\alpha \geq 1$, then the property $R^{1} f \in \mathcal{K}_{\alpha+1}(n)$ implies that $f \in \mathcal{K}_{\alpha}(n)$.

Proof. Let $f \in \mathcal{K}_{\alpha}(n)$, that is, $f=K_{\alpha}[\mu]$. Then

$$
f+\mathcal{R} f=\alpha K_{\alpha+1}[\mu]+(1-\alpha) f \in \mathcal{K}_{\alpha+1}(n)
$$

because $(1-\alpha) f \in \mathcal{K}_{\alpha}(n) \subset \mathcal{K}_{\alpha+1}(n)$. The proof of part (i) is complete.

We split the proof of part (ii) into two steps.

Step 1. $\alpha=m \in \mathbb{N}$. By assumption, we have

$$
R^{1} f(t z)=\int_{\partial B_{n}} \frac{1}{(1-t\langle z, \zeta\rangle)^{m+1}} d \mu(\zeta), \quad z \in B_{n}, t \in[0,1] .
$$

Hence, using (4.2), we obtain

$$
f(z)=\int_{\partial B_{n}} \int_{0}^{1} \frac{1}{(1-t\langle z, \zeta\rangle)^{m+1}} d t d \mu(\zeta), \quad z \in B_{n} .
$$

Let $I(z, \zeta)$ denote the inner integral. If $\langle z, \zeta\rangle=0$, then $I(z, \zeta)=1$. If $\langle z, \zeta\rangle \neq 0$, then

$$
I(z, \zeta)=\frac{1}{m} \frac{1-(1-\langle z, \zeta\rangle)^{m}}{\langle z, \zeta\rangle(1-\langle z, \zeta\rangle)^{m}}=\frac{1}{m} \sum_{j=1}^{m}(1-\langle z, \zeta\rangle)^{-j} .
$$

Therefore, in both cases we have

$$
I(z, \zeta)=\frac{1}{m} \sum_{j=1}^{m}(1-\langle z, \zeta\rangle)^{-j}
$$

Consequently,

$$
f(z)=\frac{1}{m} \sum_{j=1}^{m} \int_{\partial B_{n}} \frac{1}{(1-\langle z, \zeta\rangle)^{j}} d \mu(\zeta) .
$$

Finally, we see that $f \in \mathcal{K}_{m}(n)$, because $\mathcal{K}_{j}(n) \subset \mathcal{K}_{m}(n)$ for $j=1, \ldots, m-1$.

Step 2. $\alpha>1, \alpha \notin \mathbb{N}$. Repeating the arguments of Step 1, we get

$$
f(z)=\int_{\partial B_{n}} \int_{0}^{1} \frac{1}{(1-t\langle z, \zeta\rangle)^{\alpha+1}} d t d \mu(\zeta), \quad z \in B_{n} .
$$

We represent the inner integral as a sum of two summands and integrate by parts in the second summand, obtaining

$$
\begin{aligned}
\int_{0}^{1} \frac{1}{(1-t\langle z, \zeta\rangle)^{\alpha+1}} d t & =\int_{0}^{1} \frac{1}{(1-t\langle z, \zeta\rangle)^{\alpha}} d t+\int_{0}^{1} \frac{t\langle z, \zeta\rangle}{(1-t\langle z, \zeta\rangle)^{\alpha+1}} d t \\
& =\frac{1}{\alpha} \frac{1}{(1-\langle z, \zeta\rangle)^{\alpha}}+\frac{\alpha-1}{\alpha} \int_{0}^{1} \frac{1}{(1-t\langle z, \zeta\rangle)^{\alpha}} d t .
\end{aligned}
$$

Hence, Fubini's theorem yields

$$
f(z)=\frac{1}{\alpha} \int_{\partial B_{n}} \frac{1}{(1-\langle z, \zeta\rangle)^{\alpha}} d \mu(\zeta)+\frac{\alpha-1}{\alpha} \int_{0}^{1} \int_{\partial B_{n}} \frac{1}{(1-\langle t z, \zeta\rangle)^{\alpha}} d \mu(\zeta) d t .
$$

By definition, the first summand in the above sum belongs to $\mathcal{K}_{\alpha}(n)$. 
Consider the second summand. Let $[\alpha]=m \in \mathbb{N}$. Note that $m+1>\alpha$. Hence, by part (ii) of Corollary 2.3 , there exists a measure $\rho \in M(n)$ such that $K_{m+1}[\rho]=K_{\alpha}[\mu]$. Therefore, the second summand has the following form:

$$
\frac{\alpha-1}{\alpha} \int_{0}^{1} \int_{\partial B_{n}} \frac{1}{(1-\langle t z, \zeta\rangle)^{m+1}} d \rho(\zeta) d t .
$$

At Step 1 it was proved that the above function belongs to $\mathcal{K}_{m}(n) \subset \mathcal{K}_{\alpha}(n)$.

To refine Proposition 4.3, we need the following lemma.

Lemma 4.4. Assume that $\alpha>0$ and $g(w)=1-(1-w)^{\alpha}, w \in B_{1}$. Then there exists a measure $\rho \in M(1)$ such that $(1-w)^{-\alpha} g(w) / w=K_{\alpha}[\rho](w), w \in B_{1}$.

Proof. The definitions imply that $(g(w) / w)^{\prime} w^{2}=g^{\prime}(w) w-g(w) \in H^{1}\left(B_{1}\right)$, where $H^{1}\left(B_{1}\right)$ denotes the Hardy space. Note that $h \in H^{1}\left(B_{1}\right)$ if $h \in \mathcal{H}$ ol $\left(B_{n}\right)$ and $h(w) w^{2} \in$ $H^{1}\left(B_{1}\right)$. Putting $h(w)=(g(w) / w)^{\prime}$, we obtain $(g(w) / w)^{\prime} \in H^{1}\left(B_{1}\right)$. Hence, $g(w) / w \in$ $\mathfrak{M}_{\alpha}(1)$ by [16, Theorem 3.5]. Since $(1-w)^{-\alpha} \in \mathcal{K}_{\alpha}(1)$, the required measure $\rho \in M(1)$ exists by the definition of the multiplier space $\mathfrak{M}_{\alpha}(1)$.

Theorem 4.5. Assume that $\alpha>0$ and $f \in \mathcal{H o l}\left(B_{n}\right), n \in \mathbb{N}$. Then $f \in \mathcal{K}_{\alpha}(n)$ if and only if $R^{1} f \in \mathcal{K}_{\alpha+1}(n)$.

Proof. Suppose that $R^{1} f=K_{\alpha+1}[\mu]$, where $\mu \in M(n)$. Applying formula (4.2) and reversing the order of integration, we obtain

$$
\begin{aligned}
\alpha f(z) & =\int_{\partial B_{n}} \int_{0}^{1} \frac{\alpha d t}{(1-t\langle z, \zeta\rangle)^{\alpha+1}} d \mu(\zeta) \\
& =\int_{\partial B_{n}} \frac{1-(1-\langle z, \zeta\rangle)^{\alpha}}{\langle z, \zeta\rangle(1-\langle z, \zeta\rangle)^{\alpha}} d \mu(\zeta), \quad z \in B_{n},
\end{aligned}
$$

where the integrand is assumed to be equal to $\alpha$ if $\langle z, \zeta\rangle=0$. We put $w=\langle z, \zeta\rangle$ and apply Lemma 4.4. Reversing the order of integration, we have

$$
\begin{aligned}
\alpha f(z) & =\int_{\partial B_{1}} \int_{\partial B_{n}}(1-\langle z, \lambda \zeta\rangle)^{-\alpha} d \mu(\zeta) d \rho(\lambda) \\
& =\int_{\partial B_{1}} K_{\alpha}\left[\mu_{\lambda}\right](z) d \rho(\lambda), \quad z \in B_{n},
\end{aligned}
$$

where $\mu_{\lambda}(E)=\mu(\bar{\lambda} E)$ for the Borel sets $E \subset \partial B_{n}$. Note that $K_{\alpha}\left[\mu_{\lambda}\right](z)$ is a continuous function of the variable $\lambda \in \partial B_{1}$. Below we show that the last integral is in the family $\mathcal{K}_{\alpha}(n)$. Without loss of generality, we may assume that $\rho$ is a probability measure. We approximate the measure $\rho$ in the weak ${ }^{*}$ topology by the probability measures $\rho_{k}=$ $\sum_{j=1}^{J(k)} a_{k, j} \delta_{\lambda_{k, j}}$, where $\delta_{\lambda_{k, j}}$ denotes the $\delta$-measure at $\lambda_{k, j} \in \partial B_{1}$. By the definition of weak* convergence, we have

$$
\sum_{j=1}^{J(k)} a_{k, j} K_{\alpha}\left[\mu_{\lambda_{k, j}}\right](z) \stackrel{k \rightarrow \infty}{\longrightarrow} \int_{\partial B_{1}} K_{\alpha}\left[\mu_{\lambda}\right](z) d \rho(\lambda), \quad z \in B_{n} .
$$

Next, observe that the sequence $\left\{\sum_{j=1}^{J(k)} a_{k, j} \mu_{\lambda_{k, j}}\right\}$ is norm bounded. Hence, there exists a subsequence which converges in the weak* topology to a measure $\nu \in M(n)$. Therefore,

$$
\sum_{j=1}^{J(k)} a_{k, j} K_{\alpha}\left[\mu_{\lambda_{k, j}}\right](z) \stackrel{k \rightarrow \infty}{\longrightarrow} K_{\alpha}[\nu](z), \quad z \in B_{n} .
$$

Finally, we obtain $f=K_{\alpha}[\nu / \alpha]$. Now, it suffices to refer to Proposition 4.3 . 
Note that Theorem 4.5 extends to the case of $\alpha=0$ (see Corollary 5.7).

\section{§5. Families $\mathcal{K}_{\alpha}(n)$ and Bergman-Sobolev spaces}

5.1. Modified operators of fractional differentiation. Consider a pair of parameters $(\beta, t) \in \mathbb{R}^{2}$ with the property that neither $n-1-\beta$ nor $n-1-\beta+t$ is a strictly negative integer. We define an operator

$$
R^{\beta, t}: \mathcal{H o l}\left(B_{n}\right) \rightarrow \mathcal{H o l}\left(B_{n}\right)
$$

as follows. If

$$
f(z)=\sum_{k=0}^{\infty} f_{k}(z)
$$

is the homogeneous expansion of $f \in \mathcal{H}$ ol $\left(B_{n}\right)$, then

$$
R^{\beta, t} f(z)=\sum_{k=0}^{\infty} \frac{\Gamma(n-\beta) \Gamma(n+k-\beta+t)}{\Gamma(n-\beta+t) \Gamma(n+k-\beta)} f_{k}(z) .
$$

The inverse of $R^{\beta, t}$, denoted by $R_{\beta, t}$, is given by the formula

$$
R_{\beta, t} f(z)=\sum_{k=0}^{\infty} \frac{\Gamma(n-\beta+t) \Gamma(n+k-\beta)}{\Gamma(n-\beta) \Gamma(n+k-\beta+t)} f_{k}(z) .
$$

Assume that $\beta<n, t>0$, and identity (5.1) is true. Also, suppose that $r \in[0,1]$ and $z \in B_{n}$. By the definition of the operator $R^{\beta, t}$, we have

$$
\frac{\Gamma(n-\beta+t)}{\Gamma(n-\beta) \Gamma(t)} R^{\beta, t} f(r z)=\sum_{k=0}^{\infty} \frac{r^{k} f_{k}(z)}{B(n+k-\beta, t)} .
$$

Therefore,

$$
\frac{\Gamma(n-\beta+t)}{\Gamma(n-\beta) \Gamma(t)} \int_{0}^{1} R^{\beta, t} f(r z) r^{n-\beta-1}(1-r)^{t-1} d r=\sum_{k=0}^{\infty} f_{k}(z)=f(z)
$$

Straightforward calculations imply the following lemma.

Lemma 5.1 (cf. [30, Proposition 1.14]). Let $n \in \mathbb{N}$. Suppose that neither $n-1-\beta$ nor $n-1-\beta+t$ is a strictly negative integer. Then

$$
R^{\beta, t}\left(\frac{1}{(1-\langle z, \zeta\rangle)^{n-\beta}}\right)=\frac{1}{(1-\langle z, \zeta\rangle)^{n-\beta+t}}
$$

for all points $z \in B_{n}$ and $\zeta \in \partial B_{n}$.

5.2. A condition sufficient for membership in $\mathcal{K}_{\alpha}(n)$ with $\alpha>n$. For $n=1$, the following fact was proved in [13].

Proposition 5.2. Suppose $n \in \mathbb{N}$ and $\alpha>n$. If $g \in \mathcal{H}$ ol $\left(B_{n}\right)$ and

$$
\int_{0}^{1} \int_{\partial B_{n}}|g(r \zeta)|(1-r)^{\alpha-n-1} d \sigma_{n}(\zeta) d r=V<+\infty
$$

then $g \in \mathcal{K}_{\alpha}(n)$ and $\|g\|_{\mathcal{K}_{\alpha}(n)} \leq C(\alpha, n, V)=(\alpha-n) \cdots(\alpha-1) V /(n-1) !$

Proof. Put $t=\alpha-n$ and $f=R_{0, t} g$. Then $g=R^{0, t} f$ and

$$
f(z)=\frac{\Gamma(n+t)}{\Gamma(n) \Gamma(t)} \int_{0}^{1} g(r z) r^{n-1}(1-r)^{t-1} d r
$$


by (5.3). If $0<s<1$, then

$$
\begin{aligned}
\frac{\Gamma(n) \Gamma(t)}{\Gamma(n+t)} \int_{\partial B_{n}}|f(s \zeta)| d \sigma_{n}(\zeta) & \leq \int_{0}^{1} \int_{\partial B_{n}}|g(s r \zeta)| d \sigma_{n}(\zeta)(1-r)^{t-1} d r \\
& \leq \int_{0}^{1} \int_{\partial B_{n}}|g(r \zeta)| d \sigma_{n}(\zeta)(1-r)^{t-1} d r \\
& =V<+\infty
\end{aligned}
$$

Therefore, $f \in H^{1}\left(B_{n}\right) \subset \mathcal{K}_{n}(n)$ and $\|f\|_{\mathcal{K}_{n}(n)} \leq\|f\|_{H^{1}\left(B_{n}\right)} \leq C(\alpha, n, V)$. In other words,

$$
f(z)=\int_{\partial B_{n}} \frac{1}{(1-\langle z, \zeta\rangle)^{n}} d \mu(\zeta), \quad z \in B_{n},
$$

where $\|\mu\|_{M(n)} \leq C(\alpha, n, V)$. Thus, with the help of Lemma 5.1, we obtain

$$
g(z)=R^{0, t} f(z)=\int_{\partial B_{n}} \frac{1}{(1-\langle z, \zeta\rangle)^{n+t}} d \mu(\zeta), \quad z \in B_{n}
$$

Hence, $\|g\|_{\mathcal{K}_{\alpha}(n)} \leq C(\alpha, n, V)$.

5.3. Bergman-Sobolev spaces. Suppose $j \in \mathbb{N}$ and $q>0$. By definition, the Bergman-Sobolev space $A_{q, j}^{1}\left(B_{n}\right)$ consists of all functions $f \in \mathcal{H o l}\left(B_{n}\right)$ for which

$$
\int_{0}^{1} \int_{\partial B_{n}}\left|R^{j} f(r \zeta)\right|(1-r)^{q-1} d \sigma_{n}(\zeta) d r<+\infty,
$$

where $R^{j}=\left(R^{1}\right)^{j}$. For $n=1$, the following assertion coincides with [13, Lemma 2].

Proposition 5.3. Suppose $n \in \mathbb{N}, j \in\{1, \ldots, n\}$, and $\alpha>n-j$.

(i) If $f \in A_{\alpha-n+j, j}^{1}\left(B_{n}\right)$, then $f \in \mathcal{K}_{\alpha}(n)$.

(ii) Let $\beta>\alpha$. Then $\mathcal{K}_{\alpha}(n) \subset A_{\beta-n+j, j}^{1}\left(B_{n}\right)$.

Proof. We prove part (i). Since $\alpha+j>n$, Proposition 5.2 shows that $R^{j} f \in \mathcal{K}_{\alpha+j}(n)$. Since $\alpha>0$, repeated application of Theorem 4.5 guarantees that $f \in \mathcal{K}_{\alpha}(n)$.

Now, we turn to part (ii). Let $f \in \mathcal{K}_{\alpha}(n), \alpha>n-j$. By Theorem 4.5, we have $R^{j} f \in \mathcal{K}_{\alpha+j}(n)$; that is,

$$
R^{j} f(z)=\int_{\partial B_{n}} \frac{1}{(1-\langle z, \zeta\rangle)^{\alpha+j}} d \mu(\zeta), \quad z \in B_{n}
$$

for some measure $\mu \in M(n)$. Therefore, if $0 \leq r<1$, then

$$
\int_{\partial B_{n}}\left|R^{j} f(r \xi)\right| d \sigma_{n}(\xi) \leq \int_{\partial B_{n}} \int_{\partial B_{n}} \frac{1}{|1-r\langle\xi, \zeta\rangle|^{\alpha+j}} d \sigma_{n}(\xi) d|\mu|(\zeta) .
$$

Since $\alpha+j>n$, Lemma 3.1 yields

$$
\int_{\partial B_{n}}\left|R^{j} f(r \xi)\right| d \sigma_{n}(\xi) \leq C\|\mu\|_{M(n)}(1-r)^{n-\alpha-j} .
$$

Now, assume that $\beta>\alpha$. Then the above inequality guarantees that

$$
\int_{0}^{1} \int_{\partial B_{n}}\left|R^{j} f(r \xi)\right|(1-r)^{\beta-n+j-1} d \sigma_{n}(\xi) d r \leq C\|\mu\| \int_{0}^{1}(1-r)^{\beta-\alpha-1}<+\infty .
$$

In other words, $f \in A_{\beta-n+j, j}^{1}\left(B_{n}\right)$. 


\subsection{Bergman-Sobolev spaces and $\mathcal{K}_{0}(n)$.}

Lemma 5.4. Let $n \in \mathbb{N}$. Then $\mathcal{K}_{0}(n) \subset A_{\varepsilon, n}^{1}\left(B_{n}\right)$ for any $\varepsilon>0$.

Proof. Let $f \in \mathcal{K}_{0}(n)$. Recall that $\mathcal{K}_{0}(n) \subset \mathcal{K}_{n}(n)$ by part (iii) of Corollary 3.3. Next, $\mathcal{R}^{j} f \in \mathcal{K}_{j}(n) \subset \mathcal{K}_{n}(n)$ for $j=1,2, \ldots, n$ by Propositions 4.1 , 4.2 and Corollary 2.3. Hence, $R^{n} f=(I+\mathcal{R})^{n} f \in \mathcal{K}_{n}(n)$; that is,

$$
R^{n} f(z)=\int_{\partial B_{n}} \frac{1}{(1-\langle z, \zeta\rangle)^{n}} d \mu(\zeta), \quad z \in B_{n}
$$

for some measure $\mu \in M(n)$. Hence, if $0 \leq r<1$, then

$$
\int_{\partial B_{n}}\left|R^{n} f(r \xi)\right| d \sigma_{n}(\xi) \leq \int_{\partial B_{n}} \int_{\partial B_{n}} \frac{1}{|1-r\langle\xi, \zeta\rangle|^{n}} d \sigma_{n}(\xi) d|\mu|(\zeta) .
$$

By Lemma 3.1, we have

$$
\int_{\partial B_{n}}\left|R^{n} f(r \xi)\right| d \sigma_{n}(\xi) \leq C \log \frac{e}{1-r} .
$$

The above estimate implies that

$$
\int_{0}^{1} \int_{\partial B_{n}}\left|R^{n} f(r \xi)\right|(1-r)^{\varepsilon-1} d \sigma_{n}(\xi) d r \leq C \int_{0}^{1}(1-r)^{\varepsilon-1} \log \frac{e}{1-r}<+\infty,
$$

as required.

5.5. An embedding property for $\mathcal{K}_{0}(n)$. It is well known that $\mathcal{K}_{0}(1) \subset \mathcal{K}_{\alpha}(1)$ for all $\alpha>0$.

Corollary 5.5. Let $n \in \mathbb{N}$. Then $\mathcal{K}_{0}(n) \subset \mathcal{K}_{\alpha}(n)$ for all $\alpha>0$.

Proof. Lemma 5.4 guarantees that $\mathcal{K}_{0}(n) \subset A_{\alpha, n}^{1}\left(B_{n}\right), \alpha>0$. It remains to note that $A_{\alpha, n}^{1}\left(B_{n}\right) \subset \mathcal{K}_{\alpha}(n)$ by part (i) of Proposition 5.3 .

A. B. Aleksandrov observed that Corollary 5.5 can also be deduced from the embedding $\mathcal{K}_{0}(1) \subset \mathcal{K}_{\alpha}(1)$ for all $\alpha>0$.

5.6. The families $\mathcal{K}_{\alpha}(n), \alpha \geq 0$, and differentiation. We have the following refinement of Proposition 4.2 .

Corollary 5.6. Suppose $n \in \mathbb{N}$ and $\alpha \geq 0$. Then $f \in \mathcal{K}_{\alpha}(n)$ if and only if $\mathcal{R} f \in$ $\mathcal{K}_{\alpha+1}(n)$.

Proof. We apply Propositions 4.1, 4.2 and Corollary [5.5.

The next corollary refines Theorem 4.5.

Corollary 5.7. Suppose $n \in \mathbb{N}$ and $\alpha \geq 0$. Then $f \in \mathcal{K}_{\alpha}(n)$ if and only if $R^{1} f \in$ $\mathcal{K}_{\alpha+1}(n)$.

Proof. The case of $\alpha>0$ is covered by Theorem 4.5. So, assume that $f \in \mathcal{K}_{0}(n)$. Corollary 5.5 guarantees that $f \in \mathcal{K}_{1}(n)$. On the other hand, $\mathcal{R} f \in \mathcal{K}_{1}(n)$ by Corollary 5.6. Therefore, $R^{1} f=f+\mathcal{R} f \in \mathcal{K}_{1}(n)$.

To prove the reverse implication, assume that $R^{1} f \in \mathcal{K}_{1}(n)$. Since $\mathcal{K}_{1}(n) \subset \mathcal{K}_{2}(n)$, we have $R^{1} f \in \mathcal{K}_{2}(n)$. Hence, $f \in \mathcal{K}_{1}(n)$ by Theorem 4.5. Therefore, $\mathcal{R} f=R^{1} f-f \in$ $\mathcal{K}_{1}(n)$. So, $f \in \mathcal{K}_{0}(n)$ by Corollary 5.6 . 
5.7. Modified Bergman-Sobolev spaces and $\mathcal{K}_{\alpha}(n)$. Suppose $n \in \mathbb{N}, j \in \mathbb{N}$, and $q>0$. In some applications, it is convenient to use the modified Bergman-Sobolev space $\widetilde{A}_{q, j}^{1}\left(B_{n}\right)$ in place of $A_{q, j}^{1}\left(B_{n}\right)$. By definition, the space $\widetilde{A}_{q, j}^{1}\left(B_{n}\right)$ consists of functions $f \in \mathcal{H o l}\left(B_{n}\right)$ such that

$$
\begin{aligned}
\|f\|_{\widetilde{A}_{q, j}^{1}\left(B_{n}\right)} & =\sum_{|m| \leq j-1}\left|\frac{\partial^{m} f}{\partial z^{m}}(0)\right|+\int_{0}^{1} \int_{\partial B_{n}}\left|\mathcal{R}^{j} f(r \zeta)\right|(1-r)^{q-1} d \sigma_{n}(\zeta) d r \\
& <+\infty
\end{aligned}
$$

where $m=\left(m_{1}, \ldots, m_{n}\right) \in \mathbb{Z}_{+}^{n}$ and $|m|=m_{1}+\cdots+m_{n}$. The space $\widetilde{A}_{q, j}^{1}\left(B_{n}\right)$ with the norm $\|\cdot\|_{\widetilde{A}_{q, j}^{1}\left(B_{n}\right)}$ is a Banach space (cf. [30, Proposition 6.2]).

Proposition 5.8. Suppose $n \in \mathbb{N}, j \in\{1, \ldots, n\}$, and $\alpha>n-j$.

(i) The embedding $\widetilde{A}_{\alpha-n+j, j}^{1}\left(B_{n}\right) \subset \mathcal{K}_{\alpha}(n)$ holds true. If $f \in \widetilde{A}_{\alpha-n+j, j}^{1}\left(B_{n}\right)$, then $\|f\|_{\mathcal{K}_{\alpha}(n)} \leq C\|f\|_{\widetilde{A}_{\alpha-n+j, j}^{1}\left(B_{n}\right)}$, where the constant $C>0$ does not depend on $f$.

(ii) Let $\beta>\alpha$. Then $\mathcal{K}_{\alpha}(n) \subset \widetilde{A}_{\beta-n+j, j}^{1}\left(B_{n}\right)$.

Proof. Assume that $f \in \widetilde{A}_{\alpha-n+j, j}^{1}\left(B_{n}\right)$. We have

$$
\int_{0}^{1} \int_{\partial B_{n}}\left|\mathcal{R}^{j} f(r \zeta)\right|(1-r)^{\alpha+j-n-1} d \sigma_{n}(\zeta) d r<+\infty .
$$

Since $\alpha+j>n$, Proposition 5.2 yields $\mathcal{R}^{j} f \in \mathcal{K}_{\alpha+j}(n)$. Repeated application of Corollary 5.6 shows that $f \in \mathcal{K}_{\alpha}(n)$.

Now, consider the operator $I: \widetilde{A}_{\alpha-n+j, j}^{1}\left(B_{n}\right) \rightarrow \mathcal{K}_{\alpha}(n)$ defined by $I f=f$. If a functional sequence converges in the space $\widetilde{A}_{\alpha-n+j, j}^{1}\left(B_{n}\right)$ or in the space $\mathcal{K}_{\alpha}(n)$, then this sequence converges uniformly on the compact subsets of the ball $B_{n}$. Hence, the graph of the operator $I$ is closed. So, by the closed graph theorem,

$$
\|f\|_{\mathcal{K}_{\alpha}(n)} \leq C\|f\|_{\widetilde{A}_{\alpha-n+j, j}^{1}\left(B_{n}\right)}
$$

for all $f \in \widetilde{A}_{\alpha-n+j, j}^{1}\left(B_{n}\right)$. Part (i) is proved.

Part (ii). Let $f \in \mathcal{K}_{\alpha}(n), \alpha>n-j$. We have $\mathcal{R}^{j} f \in \mathcal{K}_{\alpha+j}(n)$ by Proposition 4.2. To finish the argument, it suffices to repeat the proof of part (ii) of Proposition 5.3.

5.8. Fractional Cauchy transforms and inner functions. Assume that $n \in \mathbb{N}$, $j \in \mathbb{N}$, and $q>0$. The space $\widetilde{A}_{q, j}^{2}\left(B_{n}\right)$ consists of all functions $f \in \mathcal{H o l}\left(B_{n}\right)$ such that

$$
\int_{0}^{1} \int_{\partial B_{n}}\left|\mathcal{R}^{j} f(r \zeta)\right|^{2}(1-r)^{q-1} d \sigma_{n}(\zeta) d r<+\infty
$$

Lemma 5.9. Suppose $n \in \mathbb{N}, j \in\{1, \ldots, n\}$, and $\beta>\alpha>n-j$. Then

$$
H^{\infty}\left(B_{n}\right) \cap \mathcal{K}_{\alpha}(n) \subset \widetilde{A}_{\beta-n+2 j, j}^{2}\left(B_{n}\right) .
$$

Proof. Let $f \in H^{\infty}\left(B_{n}\right) \cap \mathcal{K}_{\alpha}(n)$. Since $f \in H^{\infty}\left(B_{n}\right)$, we have

$$
f(z)=\int_{\partial B_{n}} \frac{f^{*}(\zeta)}{(1-\langle z, \zeta\rangle)^{n}} d \sigma_{n}(\zeta), \quad z \in B_{n} .
$$

Therefore,

$$
\mathcal{R} f(z)=\int_{\partial B_{n}} \frac{n\langle z, \zeta\rangle f^{*}(\zeta)}{(1-\langle z, \zeta\rangle)^{n+1}} d \sigma_{n}(\zeta), \quad z \in B_{n}
$$


Hence, using Lemma 3.1, we obtain

$$
|\mathcal{R} f(z)| \leq \int_{\partial B_{n}} \frac{C}{|1-\langle z, \zeta\rangle|^{n+1}} d \sigma_{n}(\zeta) \leq \frac{C}{(1-|z|)}, \quad z \in B_{n} .
$$

Arguing similarly, we verify that

$$
\left|\mathcal{R}^{j} f(z)\right| \leq \frac{C}{(1-|z|)^{j}}, \quad z \in B_{n} .
$$

Since $f \in \mathcal{K}_{\alpha}(n)$, Proposition 4.2 guarantees that $\mathcal{R}^{j} f \in \mathcal{K}_{\alpha+j}(n)$. Since $\alpha+j>n$ and $\beta>\alpha$, part (ii) of Proposition 5.8 yields

$$
\int_{0}^{1} \int_{\partial B_{n}}\left|\mathcal{R}^{j} f(r \zeta)\right|(1-r)^{\beta-n+j-1} d \sigma_{n}(\zeta) d r<\infty .
$$

Hence, applying inequality (5.4), we get

$$
\int_{0}^{1} \int_{\partial B_{n}}\left|\mathcal{R}^{j} f(r \zeta)\right|(1-r)^{j} \cdot\left|\mathcal{R}^{j} f(r \zeta)\right|(1-r)^{\beta-n+j-1} d \sigma_{n}(\zeta) d r<+\infty
$$

as required.

Recall that a nonconstant function $f \in H^{\infty}\left(B_{n}\right)$ is said to be inner if $\left|f^{*}\right|=1 \sigma_{n}$-a.e. On the one hand, the families $\mathcal{K}_{\alpha}(n)$ with $\alpha \geq n$ contain all inner functions because $H^{\infty}\left(B_{n}\right) \subset H^{1}\left(B_{n}\right) \subset \mathcal{K}_{n}(n) \subset \mathcal{K}_{\alpha}(n)$. On the other hand, the following assertion is true for $n \geq 2$.

Proposition 5.10. Let $n \geq 2$. If $0 \leq \alpha<n-1 / 2$, then the family $\mathcal{K}_{\alpha}(n)$ contains no inner functions.

Proof. Let $f \in \mathcal{K}_{\alpha}(n)$ be an inner function. By Corollary [5.5 and part (ii) of Corollary 2.3. there is no loss of generality in assuming that $\alpha \in(n-1, n-1 / 2)$.

Suppose $\rho \in[1 / 2,1)$ and $f_{\rho}(\zeta)=f(\rho \zeta), \zeta \in \partial B_{n}$. For $\zeta \in \partial B_{n}$ and $\lambda \in B_{1}$, put $f_{\zeta}(\lambda)=f(\lambda \zeta)$. Observe that $\mathcal{R} f(\lambda \zeta)=\lambda f_{\zeta}^{\prime}(\lambda)$; hence,

$$
\left|f^{*}(\zeta)-f_{\rho}(\zeta)\right| \leq 2 \int_{\rho}^{1}|\mathcal{R} f(r \zeta)| d r
$$

provided that $f^{*}(\zeta)$ is well defined. Applying estimate (5.5), Hölder's inequality, and Fubini's theorem, we obtain

$$
\begin{aligned}
\left\|f_{\rho}-f^{*}\right\|_{L^{2}\left(\partial B_{n}\right)}^{2} & \leq C \int_{\partial B_{n}}\left(\int_{\rho}^{1}|\mathcal{R} f(r \zeta)|(1-r)^{\frac{1}{4}}(1-r)^{-\frac{1}{4}} d r\right)^{2} d \sigma_{n}(\zeta) \\
& \leq C(1-\rho)^{\frac{1}{2}} \int_{\partial B_{n}} \int_{\rho}^{1}|\mathcal{R} f(r \zeta)|^{2}(1-r)^{\frac{1}{2}} d r d \sigma_{n}(\zeta) \\
& =o(1-\rho)^{\frac{1}{2}} \quad \text { as } \rho \rightarrow 1
\end{aligned}
$$

by Lemma 5.9. Tamm [27] proved that no inner function can satisfy the estimate $\left\|f_{\rho}-f^{*}\right\|_{L^{2}\left(\partial B_{n}\right)}^{2}=o(1-\rho)^{\frac{1}{2}}$ as $\rho \rightarrow 1-$ if $n \geq 2$. This contradiction finishes the proof of the proposition. 


\section{§6. BOUNDARY BEHAVIOR}

6.1. Directions of the maximal radial growth. The definition of the norm in the space $\mathcal{K}_{\alpha}(n), \alpha>0$, implies that

$$
|f(z)| \leq \frac{\|f\|_{\mathcal{K}_{\alpha}(n)}}{(1-|z|)^{\alpha}}, \quad z \in B_{n} .
$$

Proposition 6.1 shows that this maximal growth is possible for an at most countable set of radial directions. For $n=1$, this was proved in [11.

Suppose $\xi \in \partial B_{n}$ and $C>1$. Recall that the corresponding Korányi domain $D_{C}(\xi)$ is defined by

$$
D_{C}(\xi)=\left\{z \in B_{n}:|1-\langle z, \xi\rangle|<C(1-|z|)\right\} .
$$

Proposition 6.1. Suppose $n \in \mathbb{N}, \mu \in M(n), \alpha>0$, and $f=K_{\alpha}[\mu]$. If $\xi \in \partial B_{n}$, then

$$
\lim _{\substack{z \rightarrow \xi \\ z \in D_{C}(\xi)}}(1-\langle z, \xi\rangle)^{\alpha} f(z)=\mu(\{\xi\})
$$

for all $C>1$.

Proof. Let $\zeta, \xi \in \partial B_{n}$. We have

$$
\lim _{\substack{z \rightarrow \xi \\ z \in B_{n}}} \frac{(1-\langle z, \xi\rangle)^{\alpha}}{(1-\langle z, \zeta\rangle)^{\alpha}}=\delta_{\zeta, \xi}
$$

where $\delta_{\zeta, \xi}=0$ for $\zeta \neq \xi$, and $\delta_{\xi, \xi}=1$. If $z \in D_{C}(\xi)$, then

$$
\left|\frac{1-\langle z, \xi\rangle}{1-\langle z, \zeta\rangle}\right|^{\alpha} \leq \frac{|1-\langle z, \xi\rangle|^{\alpha}}{(1-|z|)^{\alpha}} \leq C^{\alpha}
$$

Hence, if $f=K_{\alpha}[\mu]$, then

$$
\lim _{\substack{z \rightarrow \xi \\ z \in D_{C}(\xi)}}(1-\langle z, \xi\rangle)^{\alpha} f(z)=\lim _{\substack{z \rightarrow \xi \\ z \in D_{C}(\xi)}} \int_{\partial B_{n}} \frac{(1-\langle z, \xi\rangle)^{\alpha}}{(1-\langle z, \zeta\rangle)^{\alpha}} d \mu(\zeta)=\mu(\{\xi\})
$$

by the dominated convergence theorem.

6.2. Radial behavior of functions in $\mathcal{K}_{\alpha}(n)$ with $\alpha>n$. Recall that the Bloch space $\mathfrak{B}\left(B_{n}\right)$ consists of the functions $f \in \mathcal{H o l}\left(B_{n}\right)$ such that

$$
|\mathcal{R} f(z)| \leq \frac{C}{1-|z|}, \quad z \in B_{n},
$$

for some constant $C>0$ (see [30, Chapter 3] for equivalent definitions).

Proposition 6.2. Suppose $n \in \mathbb{N}$ and $\alpha>n$. Then $\mathfrak{B}\left(B_{n}\right) \subset \mathcal{K}_{\alpha}(n)$.

Proof. Let $f \in \mathfrak{B}\left(B_{n}\right)$, that is, $|\mathcal{R} f(r \zeta)| \leq C(1-r)^{-1}$ for all $\zeta \in \partial B_{n}$ and $r \in[0,1)$. The assumption $\alpha>n$ guarantees that

$$
\int_{0}^{1} \int_{\partial B_{n}}|\mathcal{R} f(r \zeta)|(1-r)^{(\alpha+1)-n-1} d \sigma_{n}(\zeta) d r<+\infty .
$$

Since $\alpha+1>n$, we have $\mathcal{R} f \in \mathcal{K}_{\alpha+1}(n)$ by Proposition 5.2. Finally, applying Corollary [5.6. we obtain $f \in \mathcal{K}_{\alpha}(n)$.

Corollary 6.3. Let $n \in \mathbb{N}$. Then there exists a function $f \in \bigcap_{\alpha>n} \mathcal{K}_{\alpha}(n)$ such that the finite limit $\lim _{r \rightarrow 1-} f(r \zeta)$ fails to exist for all $\zeta \in \partial B_{n}$.

Proof. Ullrich 28, constructed a function $f \in \mathfrak{B}\left(B_{n}\right)$ with no finite radial limits. It remains to apply Proposition 6.2. 
6.3. Exceptional sets and Hausdorff contents: definitions. Let $0<\tau \leq 1$, and let $\zeta \in \partial B_{n}$. Put

$$
D_{\tau, C}(\zeta)=\left\{z \in B_{n}:|1-\langle z, \zeta\rangle|<C(1-|z|)^{\tau}\right\},
$$

where $C>1$ for $\tau=1$, and $C>0$ for $\tau \in(0,1)$. Note that the sets $D_{1, C}(\zeta)$ coincide with the Korányi domains $D_{C}(\zeta)$, which were considered in Subsection 6.1. Also, note that the order of contact between the set $D_{\tau, C}(\zeta)$ and the sphere $\partial B_{n}$ increases as the parameter $\tau$ decreases.

Consider a function $f: B_{n} \rightarrow \mathbb{C}$. By definition, the set $E_{\tau, C}(f)$ consists of all $\zeta \in \partial B_{n}$ for which the function $f(z)$ fails to have a limit at the point $\zeta$ as $z$ approaches $\zeta$ through $D_{\tau, C}(\zeta)$. The sets $E_{1, C}(f)$ are said to be exceptional; the sets $E_{\tau, C}(f), 0<\tau<1$, are tangentially exceptional.

To estimate the size of the sets $E=E_{\tau, C}(f)$ for $f \in \mathcal{K}_{\alpha}(n)$, it is natural to apply the nonisotropic Hausdorff contents, which are defined as follows:

$$
H_{m}(E)=H_{m}\left(E, \partial B_{n}\right)=\inf \left\{\sum_{k} \delta_{k}^{m}: E \subset \bigcup_{k} Q\left(\zeta_{k}, \delta_{k}\right)\right\},
$$

where $\zeta_{k} \in \partial B_{n}$ and $Q(\zeta, \delta)=\left\{\xi \in \partial B_{n}:|1-\langle\zeta, \xi\rangle|<\delta\right\}$.

6.4. Hardy-Sobolev spaces. Assume that a function $f \in \mathcal{H o l}\left(B_{n}\right)$ has homogeneous expansion $f=\sum_{k} f_{k}$. Then the fractional derivative of order $\beta>0$ is defined by the formula

$$
R^{\beta} f(z)=\sum_{k}(k+1)^{\beta} f_{k}(z), \quad z \in B_{n} .
$$

For $\beta>0$ and $0<p<\infty$, the Hardy-Sobolev spaces are defined by

$$
H_{\beta}^{p}\left(B_{n}\right)=\left\{f \in \mathcal{H} \text { ol }\left(B_{n}\right): \sup _{0<r<1} \int_{\partial B_{n}}\left|R^{\beta} f(r \zeta)\right|^{p} d \sigma_{n}(\zeta)<\infty\right\} .
$$

Theorem 6.4 (Ahern and Cohn [1]). Let $n-\beta p \geq 0$. If $f \in H_{\beta}^{p}\left(B_{n}\right)$, then

$$
H_{n-\beta p+\varepsilon}(E(f))=0
$$

for all $\varepsilon>0$.

Theorem 6.5 (Cascante and Ortega [8, Corollary 2.1]; see also [26]). Assume that $n \in \mathbb{N}, 0<p<\infty, 0<\beta<n / p, 0<\tau<1$, and $m=(n-\beta p) / \tau$. If $f \in H_{\beta}^{p}\left(B_{n}\right)$, then $H_{m}\left(E_{\tau, C}(f)\right)=0$ for all $C>0$.

6.5. Exceptional sets. By Corollary 6.3, there exists a function $f \in \bigcap_{\alpha>n} \mathcal{K}_{\alpha}(n)$ such that $E_{1, C}(f)=\partial B_{n}$ for all $C>1$. On the other hand, if $0 \leq \alpha \leq n$, then $\mathcal{K}_{\alpha}(n) \subset$ $H^{p}\left(B_{n}\right)$ for all $0<p<1$. Hence, if $f \in \mathcal{K}_{\alpha}(n), 0 \leq \alpha \leq n$, then $\sigma_{n}\left(E_{1, C}(f)\right)=0$ for all $C>1$. This observation can be refined in the case where $0 \leq \alpha<n$. For the spaces $\mathcal{K}_{\alpha}(1), 0 \leq \alpha<1$, similar refinements in terms of the Bessel capacities were obtained in [12].

Proposition 6.6. Suppose $n \in \mathbb{N}$ and $\alpha \in(0, n)$. If $f \in \mathcal{K}_{\alpha}(n)$, then $H_{\alpha+\varepsilon}\left(E_{1, C}(f)\right)=0$ for all $\varepsilon>0$ and $C>1$.

Proof. Let $n-\alpha>\varepsilon>0$. Then $\mathcal{K}_{\alpha}(n) \subset A_{\alpha+\varepsilon, n}^{1}\left(B_{n}\right) \subset H_{n-\alpha-\varepsilon}^{1}\left(B_{n}\right)$ by Proposition 5.3 and by [4, Theorem 5.12], respectively. It remains to apply Theorem 6.4

The following example corresponds to Proposition 6.6

Proposition 6.7. Suppose $n \in \mathbb{N}, \alpha \in(0, n), 0 \leq m<\alpha, E \subset \partial B_{n}$ is a compact set, $H_{m}(E)=0$, and $C>1$. Then $E=E_{1, C}(f)$ for some function $f \in \mathcal{K}_{\alpha}(n)$. 
Proof. Fix $p_{0}>1$ such that $m=n-(n-\alpha) p_{0}$. We have $H_{m}(E)=0$; hence, Theorem 1.2 in [1] guarantees that $E=E_{1, C}(f)$ for some function $f \in H_{n-\alpha}^{p_{0}}\left(B_{n}\right)$. Next, recall that

$$
\mathcal{K}_{\alpha}^{p_{0}}(n)=\left\{K_{\alpha}\left[g \sigma_{n}\right]: g \in L^{p_{0}}\left(\sigma_{n}\right)\right\} .
$$

By [8, Theorem 2.1], we have $H_{n-\alpha}^{p_{0}}\left(B_{n}\right)=\mathcal{K}_{\alpha}^{p_{0}}(n)$. It remains to note that $\mathcal{K}_{\alpha}^{p_{0}}(n) \subset$ $\mathcal{K}_{\alpha}(n)$.

6.6. Tangentially exceptional sets. For the spaces $\mathcal{K}_{\alpha}(1), 0 \leq \alpha<1$, tangentially exceptional sets were investigated in [10. In the case of an arbitrary dimension $n \in \mathbb{N}$, the following assertion holds true.

Proposition 6.8. Suppose $n \in \mathbb{N}, \alpha \in(0, n), \tau \in(\alpha / n, 1)$, and $m>\alpha / \tau$. If $f \in \mathcal{K}_{\alpha}(n)$, then $H_{m}\left(E_{\tau, C}(f)\right)=0$ for all $C>0$.

Proof. We have $\mathcal{K}_{\alpha}(n) \subset H_{n-\alpha-\varepsilon}^{1}\left(B_{n}\right)$ for $n-\alpha>\varepsilon>0$. It remains to apply Theorem 6.5.

The following example corresponds to Proposition 6.8

Proposition 6.9. Suppose $n \in \mathbb{N}, \alpha \in(0, n), \tau \in[\alpha / n, 1), m<\alpha / \tau$, and $C>0$. If $E \subset \partial B_{n}$ is a compact set and $H_{m}(E)=0$, then $E=E_{\tau, C}(f)$ for some function $f \in \mathcal{K}_{\alpha}(n)$.

Proof. Since $m<\alpha / \tau$, there exists $p_{0}>1$ such that

$$
m=\left(n-(n-\alpha) p_{0}\right) / \tau \text {. }
$$

Since $p_{0}>1$ and $H_{m}(E)=0$, we have $E=E_{\tau, C}(f)$ for some function $f \in \mathcal{K}_{\alpha}^{p_{0}}(n)$; see [26. Remark after Corollary 3.11]. It remains to note that $\mathcal{K}_{\alpha}^{p_{0}}(n) \subset \mathcal{K}_{\alpha}(n)$.

\section{$\S 7$. Multipliers}

In this section, we study the spaces $\mathfrak{M}_{\alpha}(n)$ that consist of multipliers for the families $\mathcal{K}_{\alpha}(n)$. Note that multipliers for the Hardy-Sobolev spaces were investigated in the recent papers [22, 5] and [25].

7.1. Necessary conditions. For $n=1$, the results of this subsection were obtained in [16].

Lemma 7.1. Suppose $n \in \mathbb{N}, \alpha>0$, and $f \in \mathfrak{M}_{\alpha}(n)$. Then $f \in H^{\infty}\left(B_{n}\right)$; moreover, $\|f\|_{H^{\infty}\left(B_{n}\right)} \leq\|f\|_{\mathfrak{M}_{\alpha}(n)}$.

Proof. Fix a point $\zeta \in \partial B_{n}$ and fix a constant $K$ for which $\|f\|_{\mathfrak{M}_{\alpha}(n)}<K$. Since $\left\|(1-\langle\cdot, \zeta\rangle)^{-\alpha}\right\|_{\mathcal{K}_{\alpha}(n)}=1$, there exists a measure $\mu_{\zeta} \in M(n)$ such that $\left\|\mu_{\zeta}\right\|_{M(n)}<K$ and

$$
\frac{f(z)}{(1-\langle z, \zeta\rangle)^{\alpha}}=\int_{\partial B_{n}} \frac{1}{(1-\langle z, \xi\rangle)^{\alpha}} d \mu_{\zeta}(\xi)
$$

In other words,

$$
f(z)=\int_{\partial B_{n}}\left(\frac{1-\langle z, \zeta\rangle}{1-\langle z, \xi\rangle}\right)^{\alpha} d \mu_{\zeta}(\xi) .
$$

Put $z=r \zeta, 0 \leq r<1$. Then

$$
|f(r \zeta)| \leq \int_{\partial B_{n}}\left(\frac{1-r}{1-r|\langle\zeta, \xi\rangle|}\right)^{\alpha} d \mu_{\zeta}(\xi) \leq\left\|\mu_{\zeta}\right\|_{M(n)}<K .
$$

Since the point $\zeta \in \partial B_{n}$ and the constant $K>\|f\|_{\mathfrak{M}_{\alpha}(n)}$ are arbitrary, we have $\|f\|_{H^{\infty}\left(B_{n}\right)} \leq\|f\|_{\mathfrak{M}_{\alpha}(n)}$. 
Lemma 7.2. Suppose $n \in \mathbb{N}, \alpha>0$, and $f \in \mathfrak{M}_{\alpha}(n)$. Then $f \in \mathcal{K}_{\alpha}(n)$; moreover, $\|f\|_{\mathcal{K}_{\alpha}(n)} \leq\|f\|_{\mathfrak{M}_{\alpha}(n)}$.

Proof. Put $I(z)=1$ for $z \in B_{n}$. Recall that $I=K_{\alpha}\left[\sigma_{n}\right]$. Since $\sigma_{n}$ is a positive measure, we have $\|I\|_{\mathcal{K}_{\alpha}(n)}=\left\|\sigma_{n}\right\|=1$. The assumption $f \in \mathfrak{M}_{\alpha}(n)$ implies that $f=f I \in \mathcal{K}_{\alpha}(n)$. Moreover, $\|f\|_{\mathcal{K}_{\alpha}(n)}=\|f I\|_{\mathcal{K}_{\alpha}(n)} \leq\|f\|_{\mathfrak{M}_{\alpha}(n)}\|I\|_{\mathcal{K}_{\alpha}(n)}=\|f\|_{\mathfrak{M}_{\alpha}(n)}$.

Proposition 7.3. Let $\alpha>0$. Then there exists a constant $C_{\alpha}$ such that, for any $f \in \mathfrak{M}_{\alpha}(n)$ and any $n \in \mathbb{N}$, the radial variation in the direction $\xi \in \partial B_{n}$ satisfies the estimate $V(f, \xi) \leq C_{\alpha}\|f\|_{\mathfrak{M}_{\alpha}}$ for all $\xi \in \partial B_{n}$.

Proof. Suppose $f \in \mathfrak{M}_{\alpha}(n), \xi \in \partial B_{n}$, and $\varepsilon>0$. By the definition of the space $\mathfrak{M}_{\alpha}(n)$, there exists a measure $\mu_{\xi}$ such that $\left\|\mu_{\xi}\right\|_{M(n)} \leq\|f\|_{\mathfrak{M}_{\alpha}(n)}+\varepsilon$ and

$$
f(z)=\int_{\partial B_{n}} \frac{(1-\langle z, \xi\rangle)^{\alpha}}{(1-\langle z, \zeta\rangle)^{\alpha}} d \mu_{\xi}(\zeta), \quad z \in B_{n} .
$$

Let $\lambda \in B_{1}$. We put $z=\lambda \xi$ and $f_{\xi}(\lambda)=f(\lambda \xi)$. Then

$$
\begin{aligned}
& f_{\xi}(\lambda)=\int_{\partial B_{n}} \frac{(1-\lambda)^{\alpha}}{(1-\lambda\langle\xi, \zeta\rangle)^{\alpha}} d \mu_{\xi}(\zeta), \\
& f_{\xi}^{\prime}(\lambda)=\alpha \int_{\partial B_{n}} \frac{(1-\lambda)^{\alpha-1}(\langle\xi, \zeta\rangle-1)}{(1-\lambda\langle\xi, \zeta\rangle)^{\alpha+1}} d \mu_{\xi}(\zeta) .
\end{aligned}
$$

Now, set $\lambda=r, 0 \leq r<1$. Fubini's theorem guarantees that

$$
\int_{0}^{1}\left|f_{\xi}^{\prime}(r)\right| d r \leq \alpha \int_{\partial B_{n}} \int_{0}^{1} \frac{(1-r)^{\alpha-1}|1-\langle\xi, \zeta\rangle|}{|1-r\langle\xi, \zeta\rangle|^{\alpha+1}} d r d\left|\mu_{\xi}\right|(\zeta) .
$$

Put $w=\langle\xi, \zeta\rangle$ and $b=|1-\langle\xi, \zeta\rangle|$. Observe that

$$
|1-r w|^{2}=(1-r)\left(1-r|w|^{2}\right)+r|1-w|^{2} \geq(1-r)^{2}+r^{2} b^{2} .
$$

Hence,

$$
\int_{0}^{1} \frac{(1-r)^{\alpha-1} b}{|1-r\langle\xi, \zeta\rangle|^{\alpha+1}} d r \leq \int_{0}^{1} \frac{(1-r)^{\alpha-1} b}{\left((1-r)^{2}+r^{2} b^{2}\right)^{(\alpha+1) / 2}} d r:=I(\alpha, b) .
$$

If $b=0$, then the proof is finished. Otherwise, as was shown in the proof of Theorem 2.6 in 16, we have $I(\alpha, b) \leq C_{\alpha}<\infty$. Therefore, estimate (7.1) implies that $V(f, \xi) \leq$ $C_{\alpha}\|f\|_{\mathfrak{M}_{\alpha}(n)}$. The proof of the proposition is finished.

Recall that a bounded nonconstant function $f \in \mathcal{H o l}\left(B_{n}\right)$ is said to be inner if $\left|f^{*}\right|=1$ $\sigma_{n}$-a.e. A complete description of the inner functions belonging to $\mathfrak{M}_{1}(1)$ was obtained in 18. For arbitrary $\alpha>0$, the inner functions in the family $\mathfrak{M}_{\alpha}(1)$ were investigated in 13. For $n \geq 2$, the answer to the corresponding question follows from Proposition 7.3 .

Corollary 7.4. Assume that $n \geq 2$ and $\alpha>0$. If $f$ is an inner function in the ball $B_{n}$, then $f \notin \mathfrak{M}_{\alpha}(n)$.

Proof. For $n \geq 2$, it is well known that any inner function has no radial limits on a dense subset of the sphere $\partial B_{n}$; see [24, $\left.\S 1\right]$. 
7.2. Sufficient conditions. Recall that $A\left(B_{n}\right)$ denotes the ball algebra. If $f \in A\left(B_{n}\right)$, then put $f^{*}=\left.f\right|_{\partial B_{n}}$. The complex modulus of continuity of the function $f^{*}$ is defined by

$$
\omega_{\mathbb{C}}\left(f^{*}, \delta\right)=\sup _{\zeta, \xi \in \partial B_{n}}\left\{\left|f^{*}(\zeta)-f^{*}(\xi)\right|: d(\zeta, \xi) \leq \delta\right\},
$$

where $\delta \in(0,2]$ and $d(\zeta, \xi)=|1-\langle\zeta, \xi\rangle|$. Note that $d(\zeta, \xi)$ is a quasimetric on the sphere.

Let $\nu_{n}$ denote the Lebesgue measure on $B_{n}$ normalized by the condition $\nu_{n}\left(B_{n}\right)=1$. Suppose that $n \geq 2$. For a Borel function $f: B_{1} \rightarrow \mathbb{C}$, it is well known that the identity

$$
\int_{\partial B_{n}} f(\langle\xi, \zeta\rangle) d \sigma_{n}(\xi)=(n-1) \int_{B_{1}} f(z)\left(1-|z|^{2}\right)^{n-2} d \nu_{1}(z)
$$

is fulfilled if its left-hand side or right-hand side is well defined.

An analog of the following statement for $n=1$ was proved in [29].

Proposition 7.5. Let $\alpha \geq n \geq 2$. Suppose that a function $g \in A\left(B_{n}\right)$ satisfies the following condition:

$$
(n-1) \int_{B_{1}} \frac{\left(1-|z|^{2}\right)^{n-2} \omega_{\mathbb{C}}\left(g^{*},|1-z|\right)}{|1-z|^{n}} d \nu_{1}(z)=C_{\omega}<+\infty .
$$

Then $g \in \mathfrak{M}_{\alpha}(n)$.

Proof. By Lemma2.4 and Proposition 2.5, it suffices to verify that the function $g$ satisfies condition (2.5) with $\alpha=n$.

Fix a point $\zeta \in \partial B_{n}$. Then

$$
\frac{g(w)}{(1-\langle w, \zeta\rangle)^{n}}=\frac{g(w)-g(\zeta)}{(1-\langle w, \zeta\rangle)^{n}}+\frac{g(\zeta)}{(1-\langle w, \zeta\rangle)^{n}}:=h_{1}(w)+h_{2}(w), \quad w \in B_{n} .
$$

Observe that $\left\|h_{2}\right\|_{\mathcal{K}_{n}(n)} \leq\|g\|_{A\left(B_{n}\right)}$.

On the other hand, we have $g(\cdot)-g(\zeta) \in H^{\infty}\left(B_{n}\right)$ and $(1-\langle\cdot, \zeta\rangle)^{-n} \in H^{p}\left(B_{n}\right)$ for all $0<p<1$. Hence, $h_{1} \in H^{p}\left(B_{n}\right)$ for all $0<p<1$. Let $h_{1}^{*}(\xi)=\lim _{r \rightarrow 1-} h_{1}(r \xi), \xi \in \partial B_{n}$, $\xi \neq \zeta$. Formula (7.2) and condition (7.3) show that $\left\|h^{*}\right\|_{L^{1}\left(\partial B_{n}\right)} \leq C_{\omega}$. Therefore, $h_{1} \in H^{1}\left(B_{n}\right)$; also, we obtain $\left\|h_{1}\right\|_{\mathcal{K}_{n}(n)} \leq\left\|h_{1}\right\|_{H^{1}\left(B_{n}\right)} \leq C_{\omega}$. This proves (2.5).

Assume that $0<\beta<1$. By definition, the standard Lipschitz space $\Lambda^{\beta}\left(\partial B_{n}\right)$ consists of all functions $f: \partial B_{n} \rightarrow \mathbb{C}$ such that

$$
|f(\zeta)-f(\xi)| \leq C_{f}|\zeta-\xi|^{\beta}
$$

for all $\zeta, \xi \in \partial B_{n}$. In other words, $\Lambda^{\beta}\left(\partial B_{n}\right)$ are the Lipschitz spaces with respect to the Euclidean metric on the sphere. Elements of these spaces can be used to construct examples of functions belonging to families $\mathfrak{M}_{\alpha}(n)$ with $\alpha \geq n$.

Corollary 7.6. Suppose $n \geq 2,0<\beta<1 / 2$, and $f \in \Lambda^{\beta}\left(\partial B_{n}\right)$. Then $K_{n}[f] \in \mathfrak{M}_{\alpha}(n)$ for $\alpha \geq n$.

Proof. Let $f \in \Lambda^{\beta}\left(\partial B_{n}\right)$. It is well known that $\omega_{\mathbb{C}}\left(K_{n}[f]^{*}, \delta\right)=\mathcal{O}\left(\delta^{\beta}\right)$ (see [2]). Hence, condition (7.3) is satisfied.

Explicit examples of functions belonging to $\mathfrak{M}_{\alpha}(n), \alpha \geq 0$, can be obtained with the help of the following assertion.

Proposition 7.7. Suppose $n \in \mathbb{N}, j \in\{0,1, \ldots, n\}$, and $\alpha \geq n-j$. Also, suppose that the derivative $\mathcal{R}^{j} g \in A\left(B_{n}\right)$ satisfies condition (7.3). Then $g \in \mathfrak{M}_{\alpha}(n)$.

Proof. We argue by induction. As the base of induction, we take the case of $j=0$. Then the claim is true by Proposition 7.5 . 
Now, let $k \in\{0,1, \ldots, n-1\}$. Assume that the required implication is proved for $j=k$.

Suppose $j=k+1 \in\{1, \ldots, n\}$ and $f \in \mathcal{K}_{\alpha}(n), \alpha \geq n-j$. We must prove that $f g \in \mathcal{K}_{\alpha}(n)$. Since $\alpha \geq n-j \geq 0$, Corollary [5.6 guarantees that $f g \in \mathcal{K}_{\alpha}(n)$ if and only if $\mathcal{R}(f g) \in \mathcal{K}_{\alpha+1}(n)$. Note that

$$
\mathcal{R}(f g)=f \mathcal{R} g+g \mathcal{R} f
$$

The function $\mathcal{R}^{j-1}(\mathcal{R} g) \in A\left(B_{n}\right)$ satisfies condition (7.3). We have $\alpha+1 \geq n-(j-1)$; hence, $\mathcal{R} g \in \mathfrak{M}_{\alpha+1}(n)$ by the inductive hypothesis. Since $f \in \mathcal{K}_{\alpha}(n) \subset \mathcal{K}_{\alpha+1}(n)$, we obtain $f \mathcal{R} g \in \mathcal{K}_{\alpha+1}(n)$ by the definition of the multiplier space.

On the other hand, $f \in \mathcal{K}_{\alpha}(n)$ if and only if $\mathcal{R} f \in \mathcal{K}_{\alpha+1}(n)$. Next, the function $\mathcal{R}^{j-1} g \in A\left(B_{n}\right)$ satisfies condition (7.3). By the inductive hypothesis, we have $g \in$ $\mathfrak{M}_{\alpha+1}(n)$; thus, $g \mathcal{R} f \in \mathcal{K}_{\alpha+1}(n)$.

Therefore, $\mathcal{R}(f g) \in \mathcal{K}_{\alpha+1}(n)$ and $f g \in \mathcal{K}_{\alpha}(n)$. The induction step is finished, and the proposition is proved.

By using the family $\mathcal{K}_{0}(n)$, other examples of multipliers can be obtained.

Proposition 7.8. Suppose $n \in \mathbb{N}, 0<\alpha<n$, and $g \in \mathcal{K}_{0}(n) \cap \mathfrak{M}_{n}(n)$. Then $g \in \mathfrak{M}_{\alpha}(n)$.

Proof. We argue by induction. As the base of induction, we take the statement that $g \in \mathfrak{M}_{\alpha}(n)$ for $\alpha \geq n$, which is true by assumption.

Now, suppose that $j \in\{0,1, \ldots, n-1\}$. Assume that the required implication is valid for $\alpha \geq n-j$. We prove that $g \in \mathfrak{M}_{\alpha}(n)$ if $\alpha>0$ and $\alpha \geq n-j-1$.

Let $f \in \mathcal{K}_{\alpha}(n)$. We must check that $f g \in \mathcal{K}_{\alpha}(n)$.

The condition $g \in \mathcal{K}_{0}(n)$ implies that $\mathcal{R} g \in \mathcal{K}_{1}(n)$. Since $\alpha>0$, we obtain $f \mathcal{R} g \in$ $\mathcal{K}_{\alpha}(n) \cdot \mathcal{K}_{1}(n) \subset \mathcal{K}_{\alpha+1}(n)$. On the other hand, $\mathcal{R} f \in \mathcal{K}_{\alpha+1}(n)$ because $f \in \mathcal{K}_{\alpha}(n)$. Next, we have $g \in \mathfrak{M}_{n-j}(n)$ by the inductive hypothesis. Also, $\mathfrak{M}_{n-j}(n) \subset \mathfrak{M}_{\alpha+1}(n)$ because $n-j \leq \alpha+1$. Thus, $g \mathcal{R} f \in \mathfrak{M}_{\alpha+1}(n) \cdot \mathcal{K}_{\alpha+1}(n) \subset \mathcal{K}_{\alpha+1}(n)$.

Therefore, $\mathcal{R}(f g)=f \mathcal{R} g+g \mathcal{R} f \in \mathcal{K}_{\alpha+1}(n)$ and $f g \in \mathcal{K}_{\alpha}(n)$ by Corollary 5.6. The induction step is finished. The proof of the proposition is complete.

Corollary 7.9. Suppose $n \in \mathbb{N}$ and $0<\alpha<n$. Also, suppose that a function $g \in$ $\mathcal{K}_{0}(n) \cap A\left(B_{n}\right)$ satisfies condition (7.3). Then $g \in \mathfrak{M}_{\alpha}(n)$.

Proof. It suffices to apply Propositions 7.5 and 7.8 .

\section{ACKNOWLEDGMENT}

The author is grateful to A. B. Aleksandrov for suggested improvements and useful comments.

\section{REFERENCES}

[1] P. Ahern and W. Cohn, Exceptional sets for Hardy-Sobolev functions, $p>1$, Indiana Univ. Math. J. 38 (1989), no. 2, 417-453. MR.0997390 (90m:32013)

[2] P. Ahern and R. Schneider, A smoothing property of the Henkin and Szegő projections, Duke Math. J. 47 (1980), no. 1, 135-143. MR0563371 (80i:32015)

[3] A. B. Aleksandrov, Function theory in the ball, Itogi Nauki i Tekhniki, Sovrem. Probl. Mat. Fund. Naprav., vol. 8, VINITI, Moscow, 1985, pp. 115-190; English transl., Encyclopaedia Math. Sci., vol. 8, Springer-Verlag, Berlin, 1994, pp. 107-178. MR0850487 (88b:32002)

[4] F. Beatrous and J. Burbea, Holomorphic Sobolev spaces on the ball, Diss. Math. (Rozprawy Mat.) 276 (1989), 60 pp. MR1010151 (90k:32010)

[5] _ On multipliers for Hardy-Sobolev spaces, Proc. Amer. Math. Soc. 136 (2008), no. 6, 21252133. MR 2383518 (2009c:32007)

[6] J. Bergh and J. Löfström, Interpolation spaces. An introduction, Grundlehren Math. Wiss., Bd. 223, Springer-Verlag, Berlin, 1976. MR0482275 (58:2349) 
[7] L. Brickman, D. J. Hallenbeck, T. H. MacGregor, and D. R. Wilken, Convex hulls and extreme points of families of starlike and convex mappings, Trans. Amer. Math. Soc. 185 (1973), 413-428. MR 0338337 (49:3102)

[8] C. Cascante and J. M. Ortega, Tangential-exceptional sets for Hardy-Sobolev spaces, Illinois J. Math. 39 (1995), no. 1, 68-85. MR.1299649 (95j:32009)

[9] J. A. Cima, A. L. Matheson, and W. T. Ross, The Cauchy transform, Math. Surveys Monogr., vol. 125, Amer. Math. Soc., Providence, RI, 2006. MR2215991 (2006m:30003)

[10] D. J. Hallenbeck, Tangential limits of Cauchy-Stieltjes transforms, Complex Variables Theory Appl. 33 (1997), no. 1-4, 129-136. MR1624915 (99b:31001)

[11] D. J. Hallenbeck and T. H. MacGregor, Growth and zero sets of analytic families of Cauchy-Stieltjes integrals, J. Anal. Math. 61 (1993), 231-259. MR.1253443 (95d:30078)

[12] _ Radial limits and radial growth of Cauchy-Stieltjes transforms, Complex Variables Theory Appl. 21 (1993), no. 3-4, 219-229. MR1276578 (95d:30077)

[13] D. J. Hallenbeck, T. H. MacGregor, and K. Samotij, Fractional Cauchy transforms, inner functions and multipliers, Proc. London Math. Soc. (3) 72 (1996), no. 1, 157-187. MR.1357091 (96j:30056)

[14] V. P. Havin, On analytic functions representable by an integral of Cauchy-Stieltjes type, Vestnik Leningrad. Univ. Mat. Mekh. Astronom. 1958, vyp. 1, 66-79. (Russian) MR0095256 (20:1762)

[15] _ Relations between certain classes of functions regular in the unit circle, Vestnik Leningrad. Univ. Mat. Mekh. Astronom. 1962, vyp. 1, 102-111. (Russian) MR0152660 (27:2635)

[16] R. A. Hibschweiler and T. H. MacGregor, Multipliers of families of Cauchy-Stieltjes transforms, Trans. Amer. Math. Soc. 331 (1992), no. 1, 377-394. MR.1120775 (93g:30057)

[17] _ Fractional Cauchy transforms, Chapman \& Hall/CRC Monogr. Surveys in Pure Appl. Math., vol. 136, Chapman \& Hall/CRC, Boca Raton, FL, 2006. MR2189294 (2007b:30001)

[18] S. V. Hruščev and S. A. Vinogradov, Inner functions and multipliers of Cauchy type integrals, Ark. Mat. 19 (1981), no. 1, 23-42. MR0625535 (83c:30027)

[19] V. G. Krotov, Estimates for maximal operators that are connected with boundary behavior and their applications, Trudy Mat. Inst. Steklov. 190 (1989), 117-138; English transl., Proc. Steklov Inst. Math. 1992, no. 1, 123-144. MR1005342 (90h:42030)

[20] T. H. MacGregor, Analytic and univalent functions with integral representations involving complex measures, Indiana Univ. Math. J. 36 (1987), no. 1, 109-130. MR0876994 (87m:30037)

[21] A. Nagel, W. Rudin, and J. H. Shapiro, Tangential boundary behavior of functions in Dirichlet-type spaces, Ann. of Math. (2) 116 (1982), no. 2, 331-360. MR0672838(84a:31002)

[22] J. M. Ortega and J. Fàbrega, Multipliers in Hardy-Sobolev spaces, Integral Equations Operator Theory 55 (2006), no. 4, 535-560. MR2250162 (2007f:46034)

[23] W. Rudin, Function theory in the unit ball of $\mathbf{C}^{n}$, Grundlehren Math. Wiss., Bd. 241, SpringerVerlag, New York-Berlin, 1980. MR0601594 (82i:32002)

[24] N New constructions of functions holomorphic in the unit ball of $\mathbf{C}^{n}$, CBMS Regional Conf. Ser. in Math., vol. 63, Published for the Conference Board of the Mathematical Sciences, Washington, DC, Amer. Math. Soc., Providence, RI, 1986. MR0840468 (87f:32013)

[25] P. Ryan and M. Stoll, Hardy-Sobolev spaces and algebras of holomorphic functions on the unit ball in $\mathbb{C}^{n}$, Complex Var. Elliptic Equ. 53 (2008), no. 6, 565-584. MR2421818 (2009b:46111)

[26] J. Sueiro, Tangential boundary limits and exceptional sets for holomorphic functions in Dirichlettype spaces, Math. Ann. 286 (1990), no. 4, 661-678. MR.1045395 (91b:32008)

[27] M. Tamm, Sur l'image par une fonction holomorphe bornée du bord d'un domaine pseudoconvexe, C. R. Acad. Sci. Paris Sér. I Math. 294 (1982), no. 16, 537-540. MR0679938 (84g:32008)

[28] D. C. Ullrich, A Bloch function in the ball with no radial limits, Bull. London Math. Soc. 20 (1988), no. 4, 337-341. MR0940289 (89k:32009)

[29] S. A. Vinogradov, M. G. Goluzina, and V. P. Khavin, Multipliers and divisors of Cauchy-Stieltjes type integrals, Zap. Nauchn. Sem. Leningrad. Otdel. Mat. Inst. Steklov. (LOMI) 19 (1970), 55-78; English transl., Sem. in Math. Steklov Math. Inst., Leningrad, vol. 19, Consultants Bureau, New York-London, 1972, pp. 29-42. MR0291471 (45:562)

[30] K. Zhu, Spaces of holomorphic functions in the unit ball, Grad. Texts in Math., vol. 226, SpringerVerlag, New York, 2005. MR2115155 (2006d:46035)

St. Petersburg Branch, Steklov Mathematical Institute, Fontanka 27, St. Petersburg 191023, RUSSIA

E-mail address: dubtsov@pdmi.ras.ru

Received 23/NOV/2008

Translated by THE AUTHOR 